\title{
Foliation of an Asymptotically Flat End by Critical Capacitors
}

\author{
Mouhammed Moustapha Fall ${ }^{1} \cdot$ Ignace Aristide Minlend ${ }^{2} \cdot$ Jesse Ratzkin $^{3}$
}

Received: 24 April 2021 / Accepted: 1 November 2021 / Published online: 5 January 2022

(c) The Author(s) 2021

\begin{abstract}
We construct a foliation of an asymptotically flat end of a Riemannian manifold by hypersurfaces which are critical points of a natural functional arising in potential theory. These hypersurfaces are perturbations of large coordinate spheres, and they admit solutions of a certain over-determined boundary value problem involving the Laplace-Beltrami operator. In a key step we must invert the Dirichlet-to-Neumann operator, highlighting the nonlocal nature of our problem.
\end{abstract}

Keywords Over-determined problem · Foliation · Asymptotically flat ends

Mathematics Subject Classification 58J05 $\cdot 58 \mathrm{~J} 32 \cdot 58 \mathrm{~J} 37 \cdot 35 \mathrm{~N} 10 \cdot 35 \mathrm{~N} 25$

\section{Introduction}

Riemannian manifolds with asymptotically flat ends play an important role in general relativity and cosmology, and so their general properties are of great interest. In particular, it is often useful to foliate an asymptotically flat end with special surfaces. Huisken and Yau [5] famously proved one can foliate a three-dimensional, asymptotically flat end with constant mean curvature spheres. Furthermore they prove these spheres share a common center, which one can take as the physical center of mass of the system. Previously, R. Ye [14] had shown one can foliate an asymptotically flat end in any dimension $n \geq 3$ provided the mass at infinity is nonzero.

\footnotetext{
$凶 \quad$ Jesse Ratzkin

jesse.ratzkin@mathematik.uni-wuerzburg.de

Mouhammed Moustapha Fall

mouhamed.m.fall@aims-senegal.org

Ignace Aristide Minlend

ignace.minlend@univ-douala.com

1 AIMS Senegal, Mbour, Senegal

2 Faculty of Economics and Applied Management, University of Douala, Douala, Cameroon

3 Institut für Mathematik, Universität Würzburg, Würzburg, Germany
} 
Subsequently, others have found special foliations by constant expansion surfaces [8], by Willmore surfaces [7], and by isoperimetric surfaces [2]. Here we investigate surfaces which are critical points of the Newton capacity. Recall that, if $K \subset \mathbb{R}^{n}$, with $n \geq 3$, is a compact set with Lipschitz boundary, one can define its Newton capacity as

$$
\operatorname{Cap}(K)=\frac{1}{n(n-2) \omega_{n}} \inf \left\{\int_{\mathbb{R}^{n}}|\nabla u|^{2} \mathrm{~d} x: u \in H^{1}\left(\mathbb{R}^{n}\right),\left.u\right|_{K} \equiv 1\right\},
$$

where $\omega_{n}$ is the Euclidean volume of an $n$-dimensional unit ball and $H^{1}\left(\mathbb{R}^{n}\right)$ is the Sobolev space of functions with one weak derivative in $L^{2}\left(\mathbb{R}^{n}\right)$. Standard results in potential theory imply this infimum is realized by the equilibrium potential function $U_{K}$, which solves the boundary value problem

$$
\Delta_{0} U_{K}=0 \text { in } \Omega=\mathbb{R}^{n} \backslash K,\left.\quad U_{K}\right|_{\partial K}=1, \quad \lim _{|y| \rightarrow \infty} U_{K}(y)=0
$$

where $\Delta_{0}$ is the usual, flat Laplacian. Moreover, the solution to (1.2) is unique among all functions which satisfy an appropriate decay condition. It is straight-forward to generalize both (1.1) and (1.2) to the setting of a compact set $K$ in a complete, noncompact Riemannian manifold $(M, g)$ with an asymptotically flat end.

The functional Cap is not scale-invariant in Euclidean space, one should not expect it to have critical points as a domain functional. Thus it is natural to seek critical, and even extremal, domains either subject to a constraint or of a modified functional which is scale invariant. One can normalize Cap using the volume of $K$ or the surface area of $\partial K$; both choices are natural and have roots in physics and potential theory [4]. Below we will seek critical sets of a volume-normalized functional, which leads us to the over-determined boundary value problem

$$
\Delta_{0} u=0 \text { on } \mathbb{R}^{n} \backslash K,\left.\quad u\right|_{\partial K}=1, \quad \lim _{|x| \rightarrow \infty} u(x)=0,\left.\quad \frac{\partial u}{\partial \eta}\right|_{\partial K}=\Lambda,
$$

where $\Lambda$ is a constant. See Sect. A for a derivation of (1.3) as the Euler-Lagrange equation of our normalized domain functional. This computation is standard, but we include it in Appendix A for the reader's convenience.

Of course, one can also choose to normalize using the surface area of $\partial K$, which leads one to a slightly different over-determined boundary value problem, namely

$$
\Delta_{0} u=0 \text { on } \mathbb{R}^{n} \backslash K,\left.\quad u\right|_{\partial K}=1, \quad \lim _{|x| \rightarrow \infty} u(x)=0,\left.\quad \frac{\partial u}{\partial \eta}\right|_{\partial K}=\Lambda H
$$

where $H$ is the mean curvature of $\partial K$. We derive this Euler-Lagrange equation as well, even though we do not require it.

A classical theorem of Serrin [12] implies that the only solutions of the overdetermined boundary value problem (1.3) in Euclidean space are round spheres, but one expects the situation to be more complicated in a general Riemannian manifold. 
Our setting is that of a Riemannian manifold $(M, g)$ of dimension $n \geq 3$ with one asymptotically flat end. In general, the Riemannian manifold $(M, g)$ has one end that is asymptotically flat to order $\tau>0$ if there exists a compact set $K \subset M$ and a diffeomorphism

$$
\Phi: \mathbb{R}^{n} \backslash \mathbf{B} \rightarrow M \backslash K
$$

such that in these coordinates

$$
g_{i j}(y)=\delta_{i j}+\mathcal{O}\left(|y|^{-\tau}\right), \quad \partial^{k} g_{i j}(y)=\mathcal{O}\left(|y|^{-\tau-k}\right)
$$

where $\mathbf{B}$ is the unit ball in $\mathbb{R}^{n}$ and $\partial^{k}$ refers to any collection of partial derivatives of order less than on equal to $k$. For our main result, we will require the metric to decay more rapidly to the flat metric, so that

$$
g_{i j}(y)=\left(1+\sigma|y|^{1-n}\right) \delta_{i j}+h_{i j}(y), \quad\left|h_{i j}(y)\right|=\mathcal{O}\left(|y|^{-n}\right), \quad \partial^{k} h_{i j}(y)=\mathcal{O}\left(|y|^{-k-n}\right)
$$

holds for some $\sigma \in \mathbb{R}$. Our decay condition (1.5) will in particular imply the ADM mass of the flat end (which is defined in the next paragraph below) is zero.

Despite its origins in classical potential theory, capacity appears naturally in the context of the geometry associated to scalar curvature and asymptotically flat manifolds. Schoen and Yau first discussed this connection in [11]. Thereafter Bray [1] proved the inequality: if $(M, g)$ is a 3-dimensional Riemannian manifold with $R_{g} \geq 0$ and one asymptotically flat end of order $\tau>1 / 2$ then

$$
\operatorname{Cap}(\Sigma) \geq m_{\mathrm{ADM}}=\frac{1}{4\left|\mathbf{S}^{n-1}\right|} \lim _{R \rightarrow \infty} \int_{|x|=R}\left(\partial_{i} g_{i j}-\partial_{j} g_{i i}\right) \frac{x^{j}}{R} \mathrm{~d} \sigma .
$$

Most recently, J. Jauregui [6] defined a capacitory version of the mass of a threedimensional asymptotically flat end as

$$
m_{\mathrm{CV}}=\sup \limsup _{j \rightarrow \infty}\left(\left(\frac{3\left|K_{j}\right|}{4 \pi}\right)^{1 / 3}-\operatorname{Cap}\left(K_{j}\right)\right),
$$

where the outer supremum is over all possible compact exhaustions of $M$. Jauregui [6] further showed that if $(M, g)$ is a three-dimensional manifold with one asymptotically flat end and $R_{g} \geq 0$ then $m_{\mathrm{CV}} \geq m_{\mathrm{ADM}}$. Moreover, if $(M, g)$ is harmonically flat then $m_{\mathrm{CV}}=m_{\mathrm{ADM}}$.

In the same paper Jauregui conjectures that such an asymptotically flat end carries a foliation by critical points of the capacity function. Our main theorem provides some evidence towards this conjecture, at least for metrics with stronger asymptotics.

Theorem 1 Let $(M, g)$ be a Riemannian manifold of dimension $n \geq 3$ with one asymptotically flat end $M \backslash K$, parameterized as in (1.4) and (1.5). Then there exists $\rho_{0}>1$ and compact sets $K_{\rho}$ indexed by $\rho \in\left(\rho_{0}, \infty\right)$ such that the domains $\Omega_{\rho}=M \backslash K_{\rho}$ 
are critical capacitors. In other words, there exist functions $\bar{u}_{\rho}$ which solve the overdetermined boundary value problem

$$
\begin{cases}\Delta_{g} \bar{u}_{\rho}=0 & \text { in } \Omega_{\rho} \\ \bar{u}_{\rho}=1 & \text { on } \partial \Omega_{\rho}=\partial K_{\rho} \\ \lim _{|y| \rightarrow \infty} \bar{u}_{\rho}(\Phi(y))=0 & \\ \frac{\partial \bar{u}_{\rho}}{\partial \eta}=C(\rho, \sigma, n) & \text { on } \quad \partial \Omega_{\rho}=\partial K_{\rho}\end{cases}
$$

where $\eta$ is the unit interior normal to $K_{\rho}$ and

$$
C(\rho, \sigma, n)=\frac{n-2}{\rho}\left(1-\frac{(n-1)}{2 \rho^{n-1}} \sigma\right) .
$$

The hypersurfaces $\left\{\partial K_{\rho}\right\}_{\rho>\rho_{0}}$ foliate the end $M \backslash K_{\rho_{0}}$.

Our result builds naturally on earlier work, particularly that of the first and second authors [3]. More precisely, they perturb small geodesic balls to produce a family of domains $\Omega_{\rho}$, parameterized by $\rho \in\left(0, \rho_{0}\right)$, which admit solutions to the overdetermined boundary value problem

$$
\Delta_{g} u=1 \text { on } \Omega_{\rho},\left.\quad u\right|_{\partial \Omega_{\rho}}=0,\left.\quad \frac{\partial u}{\partial \eta}\right|_{\partial \Omega}=\text { constant. }
$$

In our case, the sets $K_{\rho}$ will be perturbations of large coordinate spheres, as defined by the parameterization $\Phi$ in (1.4).

We end this introduction with a brief outline of the rest of the paper. We begin by reformulating our problem in Sect. 2 to take place on a fixed set. We parameterize this reformulated problem by a radius $\rho$, a translation $\tau$, and a function $w \in \mathcal{C}^{2, \alpha}(\mathbf{S})$. Sect. 3 has some preliminary computations, such as expansions of the metric and the LaplaceBeltrami operators for our reformulated problem, as well as a study of the mapping properties of the Laplace-Beltrami operator on certain weighted function spaces in Sect. 3.3. In Sect. 4 we construct an approximate solution $v$, given in (4.1), and perturb it by a translation to the eventual solution $\widehat{u}_{\rho, \tau, w}$, given in (4.10). The function $\widehat{u}_{\rho, \tau, w}$ already satisfies most of our desired properties: it is harmonic, decays appropriately, and has constant Dirichlet data. It only remains for us to choose parameters $\rho, \tau$, and $w$ so that $\widehat{u}_{\rho, \tau, w}$ also has constant Neumann data. To correctly choose these parameters we must invert the Dirichlet-to-Neumann operator of the Laplace-Beltrami operator. We do this in two steps, first writing out an expansion of the normal derivative of $\widehat{u}_{\rho, \tau, w}$ and performing a linear analysis of this expansion in Sect. 5, and then completing our nonlinear analysis using the implicit function theorem in Sect. 6. Finally, in Sect. 7 we show that we do in fact produce a foliation of the asymptotically flat end. 


\section{Reformulation of the Problem}

In this section we reformulate our problem so that we can solve a family of PDEs on the fixed Euclidean domain $\mathbb{R}^{n} \backslash \mathbf{B}$. Intuitively, we accomplish three things with this reformulation. First, we rescale by $\rho>0$, which one should take to be large. Second, we translate the center of the ball by a small parameter $\tau \in \mathbb{R}^{n}$. Third, we deform the unit sphere $\mathbf{S}=\partial \mathbf{B}$ by a function $w \in \mathcal{C}^{2, \alpha}(\mathbf{S})$.

We should imagine $\rho$ to be large and both $w$ and $\tau$ to be small. So that our parameters are all of the same scale, we require

$$
\|w\|_{\mathcal{C}^{2, \alpha}(\mathbf{S})}=\mathcal{O}\left(\rho^{-n}\right), \quad|\tau|=\mathcal{O}\left(\rho^{-n}\right)
$$

for the remainder of the paper.

Putting all these transformations together we obtain a parameterization

$$
\Phi_{\rho, w, \tau}: \mathbb{R}^{n} \backslash \mathbf{B} \rightarrow M, \quad \Phi_{\rho, w, \tau}(x)=\Phi\left(\rho \tau+\rho x+\rho w\left(\frac{x}{|x|}\right) x\right)
$$

and let $\widehat{\Omega}_{\rho, \tau, w}=\Phi_{\rho, \tau, w}(\mathbb{R} \backslash \mathbf{B})$. Finally we can use $\Phi_{\rho, w, \tau}$ to pull problem (1.6) back to $\mathbb{R}^{n} \backslash \mathbf{B}$. Under this change of coordinates, we have now reformulated our original problem (1.6) as

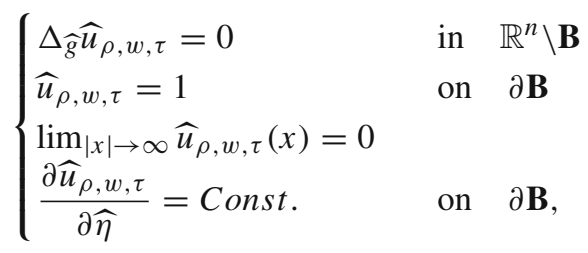

where $\widehat{g}=\Phi_{\rho, w, \tau}^{*}(g), \widehat{\eta}$ is the inward pointing unit normal to $\mathbf{S}=\partial \mathbf{B}$ with respect to the metric $\widehat{g}$. Both our new metric $\widehat{g}$ and the function $\widehat{u}_{\rho, w, \tau}$ depend on the three parameters $\rho \in(0, \infty), w \in \mathcal{C}^{2, \alpha}(\mathbf{S})$, and $\tau \in \mathbb{R}^{n}$.

\section{Preliminary Computations}

In this section we carry out some preliminary computations, in preparation for solving (2.3) We first write out a Taylor expansion of the metric $\widehat{g}$.

\subsection{Notation}

All our computations in this section are perturbation expansions, when $\rho$ is large and $|\tau|$ and $\|w\|_{\mathcal{C}^{k, \alpha}(\mathbf{S})}$ are small. In the computations below we will sometimes wish to extend a function $v$ defined on the sphere to a tubular neighborhood, and we do so by 
making it constant in the radial direction, taking $w(x)=w\left(\frac{x}{|x|}\right)$. Similarly, for each $w \in \mathcal{C}^{2, \alpha}(\mathbf{S})$ we let

$$
w_{i}\left(\frac{x}{|x|}\right)=\partial_{i} w\left(\frac{x}{|x|}\right), \quad w_{i j}\left(\frac{x}{|x|}\right)=\partial_{i j} w\left(\frac{x}{|x|}\right)
$$

and

$$
\Delta_{0} w=\sum_{i=1}^{n} w_{i i}
$$

for all $x \in \mathbb{R}^{n} \backslash\{0\}$.

To make the computations below tractable, we adopt the following notation throughout the rest of the paper.

For $i \in\{0,1,2\}$ we let $L^{i}$ denote a linear partial differential operator of order $i$ whose coefficients depend smoothly on $\rho$ and $x$ and that satisfies the bound

$$
\left\|L^{i}(v) \mid\right\|_{C^{k, \alpha}\left(\mathbb{R}^{n} \backslash \mathbf{B}\right)} \leq c\|v\|_{C^{k+i, \alpha}(\mathbf{S})}
$$

for each $v \in C^{k, \alpha}(\mathbf{S})$, where the constant $c>0$ is independent of $\rho$. Similarly we let $Q^{i}$ denote a nonlinear operator of order $i \in\{0,1,2\}$ such that $Q^{i}(0,0)=0$ and that satisfies the bound

$$
\begin{aligned}
\left\|Q^{i}\left(v_{1}, \tau_{1}\right)-Q^{i}\left(v_{2}, \tau_{2}\right)\right\|_{\mathcal{C}^{k, \alpha}(\mathbb{R} \backslash \mathbf{B})} \leq c & \left(\left\|v_{1}\right\|_{\mathcal{C}^{k+i, \alpha}(\mathbf{S})}+\left\|v_{2}\right\|_{\mathcal{C}^{k+i, \alpha}(\mathbf{S})}+\left|\tau_{1}\right|+\left|\tau_{2}\right|\right) \\
& \times\left(\left\|v_{1}-v_{2}\right\|_{\mathcal{C}^{k+i, \alpha}(\mathbf{S})}+\left|\tau_{1}-\tau_{2}\right|\right)
\end{aligned}
$$

provided $\left\|v_{1}\right\|_{C^{k+i, \alpha}(\mathbf{S})}+\left\|v_{2}\right\|_{C^{k+i, \alpha}(\mathbf{S})}+\left|\tau_{1}\right|+\left|\tau_{2}\right| \leq 1$.

Finally we let $P_{i}$ be a function of the form

$$
P_{i}(\rho, x, v, \tau)=\rho^{1-n}|x|^{1-n} L^{i}(v)+Q^{i}(v, \tau)+\mathcal{O}\left(\rho^{-n}|x|^{-n}\right)
$$

such that for every $k, \ell, m \in \mathbb{N}, x \in \mathbb{R}^{n} \backslash \mathbf{B}, \tau \in \mathbb{R}^{n}$ and $\rho>\rho_{0}>0$

$$
\left\|\partial_{\rho}^{\ell} \partial_{v}^{k} \partial_{\tau}^{m} P_{i}(\rho, \cdot, v, \tau)\right\| \leq c\left(\rho^{-n-\ell}+\rho^{1-n-\ell}\left(\|v\|_{\mathcal{C}^{i, \alpha}(\mathbf{S})}\right)+\|v\|_{\mathcal{C}^{i, \alpha}(\mathbf{S})}^{2}+|\tau|^{2}\right),
$$

for some positive constant depending only on $h, n, k, \ell, m, i, \alpha$ and $\rho_{0}$. For brevity we write

$$
P_{i}(\rho, x, v)=P_{i}(\rho, x, v, 0)
$$

It is important to observe that the product of any two terms, each of which has the form of either $L^{i}$ or $Q^{i}$, has the form of $P_{i}$. 


\subsection{Metric Expansions and the Laplacian}

We have the following expansions.

Lemma 3.1 We have

$$
\rho^{-2} \widehat{g}_{i j}(x)=\left(1+2 w+\sigma|z|^{1-n}\right) \delta_{i j}+x_{i} w_{j}+x_{j} w_{i}+P_{1}(\rho, x, w, \tau)
$$

and

$$
\rho^{2} \widehat{g}^{i j}(x)=\left(1+2 w+\sigma|z|^{1-n}\right)^{-1} \delta_{i j}-\left(x^{i} w^{j}+x^{j} w^{i}\right)+P_{1}(\rho, x, w, \tau),
$$

where

$$
|z|^{1-n}:=\rho^{1-n} r^{1-n}\left(1-\frac{n-1}{r^{2}}\langle x, \tau\rangle\right) .
$$

Proof Letting $\left\{e_{1}, \ldots, e_{n}\right\}$ be the standard orthonormal basis for $\mathbb{R}^{n}$, we see

$$
\begin{aligned}
D \Phi_{\rho, w, \tau}\left(e_{i}\right) & =\sum_{k} D \Phi\left(e_{k}\right)\left(\left(\rho+\rho w\left(\frac{x}{|x|}\right)\right) \delta_{i k}+\rho x^{k} w_{k}\right) \\
& =\rho\left(\left(1+w\left(\frac{x}{|x|}\right)\right) D \Phi\left(e_{i}\right)+\sum_{k} x^{k} w_{k} D \Phi\left(e_{k}\right)\right)
\end{aligned}
$$

where we evaluate derivatives of $\Phi$ at $\rho \tau+\rho x+\rho w(x /|x|) x$. Hence

$$
\begin{aligned}
& \rho^{-2} \widehat{g}_{i j} \\
&= g\left(\left(1+w\left(\frac{x}{|x|}\right)\right) D \Phi\left(e_{i}\right)+\sum_{k} x^{k} w_{k} D \Phi\left(e_{k}\right),\right. \\
&\left.\left(1+w\left(\frac{x}{|x|}\right)\right) D \Phi\left(e_{j}\right)+\sum_{l} x^{l} w_{l} D \Phi\left(e_{l}\right)\right) \\
&=(1+w)^{2} g_{i j}+(1+w) w_{j} x^{l} g_{i l}+(1+w) w_{i} x^{k} g_{j k}+w_{i} w_{j} x^{k} x^{l} g_{k l} .
\end{aligned}
$$

Next, we write $|x|=r$ and

$$
y=\rho\left(1+w\left(\frac{x}{|x|}\right)\right) x+\rho \tau,
$$

so that

$$
\begin{aligned}
& |y|^{2}=\rho^{2} r^{2}\left(1+2 w+\frac{2}{r^{2}}\langle x, \tau\rangle+w^{2}+2 r^{-2} w\langle x, \tau\rangle+r^{-2}|\tau|^{2}\right) \\
& |y|^{1-n}=\rho^{1-n} r^{1-n}\left(1-(n-1) w-\frac{n-1}{r^{2}}\langle x, \tau\rangle+w^{2}+r^{-2} w\langle x, \tau\rangle\right.
\end{aligned}
$$




$$
\begin{aligned}
& \left.+r^{-2}|\tau|^{2}+\cdots\right) \\
= & \rho^{1-n} r^{1-n}\left(1-\frac{n-1}{r^{2}}\langle x, \tau\rangle\right)+\rho^{1-n} r^{1-n} L^{0}(w, \tau)+Q^{0}(w, \tau) .
\end{aligned}
$$

Observe that we absorb the term $(1-n) \rho^{1-n} r^{1-n} w$ above into $L^{0}(w)$, while the corresponding linear term with respect to $\tau$ is kept. Indeed when solving the nonlinear equation for small $\rho$ in Sect. 6, we have to replace $w$ with $\rho^{n-1} w$, which increases the power of $\rho$ in $(1-n) \rho^{n-1} r^{1-n} w$ by $n-1$.

Using (1.5) and (3.1), it follows from (3.9) and (3.10) that both (3.6) and (3.7) hold.

In the next sections, we will work with the metric

$$
g_{\rho}:=\rho^{-2} \hat{g}
$$

Lemma 3.2 For $\rho$ sufficiently large,

$$
\begin{aligned}
\Delta_{g_{\rho}} & =\left(1-2 w-\sigma|z|^{1-n}\right) \Delta_{0}-\left[x_{i} w_{j}+x_{j} w_{i}+P_{1}(\rho, x, w, \tau)\right] \partial_{i j}^{2} \\
& -\left[\left(3 w_{j}-\frac{n-2}{2} \sigma \partial_{j}|z|^{1-n}\right)+x_{i} w_{i j}+\left[\Delta_{0} w\right] x_{j}+P_{2}(\rho, x, w, \tau)\right] \partial_{j},
\end{aligned}
$$

where $\Delta_{0}$ is the usual flat Laplacian.

Moreover,

$$
\begin{aligned}
& \partial_{j}|z|^{1-n}=-(n-1) \rho^{1-n} r^{-1-n} x_{j}\left(1-\frac{n-3}{r^{2}}\langle x, \tau\rangle\right) \\
& -(n-1) \rho^{1-n} r^{-1-n} \tau_{j} .
\end{aligned}
$$

Proof Recall that the Laplace-Beltrami operator has the form

$$
\Delta_{g_{\rho}} u=\frac{1}{\sqrt{\left|g_{\rho}\right|}} \partial_{i} g_{\rho}^{i j} \sqrt{\left|g_{\rho}\right|} \partial_{j} u, \quad\left|g_{\rho}\right|=\operatorname{det}\left(g_{\rho}\right) \text {. }
$$

Then using (3.6) and (3.7), we have

$$
\begin{aligned}
\left|g_{\rho}\right|= & 1+2 n w+n \sigma|z|^{1-n}+P_{1}(\rho, x, w, \tau) \\
\sqrt{\left|g_{\rho}\right|}= & 1+n w+\frac{n \sigma}{2}|z|^{1-n}+P_{1}(\rho, x, w, \tau) \\
\frac{1}{\sqrt{\left|g_{\rho}\right|}=} & 1-n w-\frac{n \sigma}{2}|z|^{1-n}+P_{1}(\rho, x, w, \tau) \\
g_{\rho}^{i j} \sqrt{\left|g_{\rho}\right|}= & {\left[\left(1+(n-2) w+\frac{n-2}{2} \sigma|z|^{1-n}\right) \delta_{i j}\right.} \\
& \left.-\left(w^{i} x^{j}+w^{j} x^{i}\right)+P_{1}(\rho, x, w, \tau)\right]
\end{aligned}
$$




$$
\begin{aligned}
& \partial_{i}\left(g_{\rho}^{i j} \sqrt{\left|g_{\rho}\right|}\right)=\left((n-3) w_{i}+\frac{n-2}{2} \sigma \partial_{i}|z|^{1-n}\right) \delta_{i j} \\
& -w_{i i} x_{j}-w_{i j} x_{i}-w_{j}+P_{2}(\rho, x, w, \tau) \\
& \frac{1}{\sqrt{\left|g_{\rho}\right|}} \partial_{i}\left(g_{\rho}^{i j} \sqrt{\left|g_{\rho}\right|}\right)=\left((n-3) w_{i}+\frac{n-2}{2} \sigma \partial_{i}|z|^{1-n}\right) \delta_{i j} \\
& -w_{i i} x_{j}-w_{i j} x_{i}-w_{j}+P_{2}(\rho, x, w, \tau) \\
& \Delta_{g_{\rho}} u=g_{\rho}^{i j} \partial_{i} \partial_{j} u+\frac{1}{\sqrt{\left|g_{\rho}\right|}} \partial_{i} \\
& \left(g_{\rho}^{i j} \sqrt{\left|g_{\rho}\right|}\right) \partial_{j} u \\
& =\left[\left(1-2 w-\sigma|z|^{1-n}\right) \delta_{i j}-\left(x^{i} w^{j}+x^{j} w^{i}\right)\right. \\
& \left.+P_{1}(\rho, x, w, \tau)\right] \partial_{i} \partial_{j} u \\
& -\left[\left(3 w_{i}-\frac{n-2}{2} \sigma \partial_{i}|z|^{1-n}\right) \partial_{i} u\right. \\
& \left.-\left(x^{j} \Delta_{0} w+x_{i} w_{i j}\right) \partial_{j} u\right]+P_{2}(\rho, x, w, \tau) \partial_{j} u .
\end{aligned}
$$

In addition we have from (3.8)

$$
\partial_{j}|z|^{1-n}=-(n-1) \rho^{1-n} r^{-1-n} x_{j}\left(1-\frac{n-3}{r^{2}}\langle x, \tau\rangle\right)-(n-1) \rho^{1-n} r^{-1-n} \tau_{j},
$$

which yield the expansions in Lemma 3.2.

\subsection{Weighted Spaces}

The best setting in which to perform our linear analysis is that of weighted Hölder spaces. Following Pacard and Rivière [9], we use the following definition.

Definition 1 Let $v \in \mathbb{R}, k \in \mathbb{N}$ and $0<\alpha<1$. Then we say $u \in \mathcal{C}_{v}^{k, \alpha}\left(\mathbb{R}^{n} \backslash \mathbf{B}\right)$ if $u \in \mathcal{C}_{\mathrm{loc}}^{k, \alpha}\left(\mathbb{R}^{n} \backslash \mathbf{B}\right)$ and

$$
\|u\|_{k, \alpha, v}=\sup _{s>1}\left(s^{-v}[u]_{k, \alpha, s}\right)<\infty
$$

Here

$$
[u]_{k, \alpha, s}:=\sum_{i=0}^{k} s^{i} \sup _{A_{s}}\left|\nabla^{i} u\right|+s^{k+\alpha} \sup _{x, x^{\prime} \in A_{s}} \frac{\left|\nabla^{k} u(x)-\nabla^{k} u\left(x^{\prime}\right)\right|}{\left|x-x^{\prime}\right|^{\alpha}}
$$

where $A_{s}=\left\{x \in \mathbb{R}^{n}: s<|x|<2 s\right\}$. We denote the space of functions vanishing on the boundary by

$$
\mathcal{C}_{\nu, \mathcal{D}}^{k, \alpha}\left(\mathbb{R}^{n} \backslash \mathbf{B}\right):=\left\{u \in \mathcal{C}_{v}^{k, \alpha}\left(\mathbb{R}^{n} \backslash \mathbf{B}\right):\left.u\right|_{\partial B}=0\right\}
$$


Intuitively, one can think of $\mathcal{C}_{v}^{0, \alpha}\left(\mathbb{R}^{n} \backslash \mathbf{B}\right)$ as those functions which grow at most like $|x|^{v}$ when $|x|$ is large.

Remark 1 Pacard and Rivière perform their analysis on weighted Hölder spaces on $\mathbf{B} \backslash\{0\}$, whereas we want to examine functions on $\mathbb{R}^{n} \backslash \mathbf{B}$. It is straight-forward to transfer between the two settings using the Kelvin transform $\mathbb{K}$, defined by

$$
\mathbb{K}: \mathcal{C}_{v}^{k, \alpha}(\mathbf{B} \backslash\{0\}) \rightarrow \mathcal{C}_{2-n-v}^{k, \alpha}\left(\mathbb{R}^{n} \backslash \mathbf{B}\right), \quad \mathbb{K}(u)(x)=|x|^{2-n} u\left(\frac{x}{|x|^{2}}\right) .
$$

It will be convenient to also note the transformation law

$$
\Delta_{0}(\mathbb{K}(u))(x)=|x|^{-4} \mathbb{K}\left(\Delta_{0} u\right)(x) .
$$

One can show the following theorem (see Sect. 2.2 of [9]).

Theorem 2 The mapping

$$
\Delta_{0}: \mathcal{C}_{v, \mathcal{D}}^{2, \alpha}\left(\mathbb{R}^{n} \backslash \mathbf{B}\right) \rightarrow \mathcal{C}_{\nu-2}^{0, \alpha}\left(\mathbb{R}^{n} \backslash \mathbf{B}\right)
$$

is injective if $v<0$ and surjective if $v>2-n$.

The mapping properties of $\Delta_{0}$ change when the weight $v$ crosses over one of the indicial roots $\gamma_{j}^{ \pm}$, where

$$
\gamma_{j}^{ \pm}=\frac{2-n}{2} \pm \sqrt{\frac{(n-2)^{2}}{4}+\lambda_{j}}
$$

and $\lambda_{j}$ is the $j$ th eigenvalue of the Laplace-Beltrami operator on the sphere. Thus one can recover the indicial roots $\gamma_{j}^{ \pm}$as growth/decay rates of solutions to the ODE

$$
w^{\prime \prime}+\frac{n-1}{r} w^{\prime}-\frac{\lambda_{j}}{r^{2}} w=0, \quad w=r^{\gamma_{j}^{ \pm}}
$$

A slightly more refined analysis uncovers the following theorem.

Theorem 3 Let $v<0$ with $v \notin\left\{\gamma_{j}^{ \pm}: j \in \mathbf{N}\right\}$, and let $j_{0}$ be the least non-negative integer such that $v>\gamma_{j_{0}}^{-}$. Then the cokernel of the mapping

$$
\Delta_{0}: \mathcal{C}_{v, \mathcal{D}}^{2, \alpha}\left(\mathbb{R}^{n} \backslash \mathbf{B}\right) \rightarrow \mathcal{C}_{v-2}^{0, \alpha}\left(\mathbb{R}^{n} \backslash \mathbf{B}\right)
$$

has dimension $j_{0}$. Alternatively let $v>2-n$ with $v \notin\left\{\gamma_{j}^{ \pm}: j \in \mathbf{N}\right\}$, and let $j_{0}$ be the least positive integer such that $v<\gamma_{j_{0}}^{+}$then kernel of the mapping

$$
\Delta_{0}: \mathcal{C}_{v, \mathcal{D}}^{2, \alpha}\left(\mathbb{R}^{n} \backslash \mathbf{B}\right) \rightarrow \mathcal{C}_{v-2}^{0, \alpha}\left(\mathbb{R}^{n} \backslash \mathbf{B}\right)
$$

has dimension $j_{0}$. 
Again, we refer the reader to Sect. 2.2 of [9] for details.

Replacing $\rho$ by $1 / \rho$ in Lemmas 3.1-3.2 and keeping the notation $g_{\rho}$ for the metric $g_{1 / \rho}$, we have the following result.

Lemma 3.3 Let $2-n<v<0$. There exist $\rho_{0}>0$ and $c_{0}>0$ such that the map

$$
\mathcal{L}:\left(0, \rho_{0}\right) \times \mathbf{B}_{c_{0}}(0) \times \mathbf{B}_{c_{0}}(0) \times \mathcal{C}_{\nu, \mathcal{D}}^{2, \alpha}\left(\mathbb{R}^{n} \backslash \mathbf{B}\right) \rightarrow \mathcal{C}_{\nu}^{0, \alpha}\left(\mathbb{R}^{n} \backslash \mathbf{B}\right)
$$

defined by

$$
\mathcal{L}(\rho, w, \tau, u):=\Delta_{g_{\rho}} u
$$

is well defined and smooth. Here $\mathbf{B}_{r}(0)$ will refer to a ball centered at the origin of radius $r$ in the appropriate space, either $\mathbb{R}^{n}$ or a space of functions depending on the context.

Furthermore, for every $(\rho, w, \tau) \in\left(0, \rho_{0}\right) \times \mathbf{B}_{c_{0}}(0) \times \mathbf{B}_{c_{0}}(0)$, the linear map

$$
\mathcal{L}_{\rho, w, \tau}: \mathcal{C}_{\nu, \mathcal{D}}^{2, \alpha}\left(\mathbb{R}^{n} \backslash \mathbf{B}\right) \rightarrow \mathcal{C}_{v}^{0, \alpha}\left(\mathbb{R}^{n} \backslash \mathbf{B}\right), \quad u \mapsto \mathcal{L}_{\rho, w, \tau}(u):=\mathcal{L}(\rho, w, \tau, u)
$$

is invertible and for all $u \in \mathcal{C}_{\nu, \mathcal{D}}^{2, \alpha}\left(\mathbb{R}^{n} \backslash \mathbf{B}\right)$ we have the inequalities

$$
C\|u\|_{\mathcal{C}_{v}^{2, \alpha}\left(\mathbb{R}^{n} \backslash \mathbf{B}\right)} \leq\left\|\mathcal{L}_{\rho, w, \tau}(u)\right\|_{\mathcal{C}_{v}^{0, \alpha}\left(\mathbb{R}^{n} \backslash \mathbf{B}\right)} \leq C^{-1}\|u\|_{\mathcal{C}_{v}^{2, \alpha}\left(\mathbb{R}^{n} \backslash \mathbf{B}\right)},
$$

where $C>0$ is independent of $(\rho, w, \tau)$.

Proof By Lemma 3.2, we can find $\rho_{0}>0$ and $c_{0}>0$ such that the map

$$
\mathcal{L}:\left(0, \rho_{0}\right) \times \mathbf{B}_{c_{0}}(0) \times \mathbf{B}_{c_{0}}(0) \times \mathcal{C}_{\nu, \mathcal{D}}^{2, \alpha}\left(\mathbb{R}^{n} \backslash \mathbf{B}\right) \rightarrow \mathcal{C}_{\nu}^{0, \alpha}\left(\mathbb{R}^{n} \backslash \mathbf{B}\right)
$$

defined by

$$
\mathcal{L}(\rho, w, \tau, u)=\Delta_{g_{\rho}} u
$$

is well defined and smooth. We shall show that the linear map

$$
u \mapsto \mathcal{L}(\rho, w, \tau, u): \mathcal{C}_{v, \mathcal{D}}^{2, \alpha}\left(\mathbb{R}^{n} \backslash \mathbf{B}\right) \rightarrow \mathcal{C}_{v}^{0, \alpha}\left(\mathbb{R}^{n} \backslash \mathbf{B}\right)
$$

is invertible for every $(\rho, w, \tau) \in\left(0, \rho_{0}\right) \times \mathbf{B}_{c_{0}}(0)$. To see this, we pick $f \in$ $\mathcal{C}_{v}^{0, \alpha}\left(\mathbb{R}^{n} \backslash \mathbf{B}\right)$ and we define

$$
\mathcal{F}:\left(-\rho_{0}, \rho_{0}\right) \times \mathbf{B}_{c_{0}}(0) \times \mathbf{B}_{c_{0}}(0) \times \mathcal{C}_{\nu, \mathcal{D}}^{2, \alpha}\left(\mathbb{R}^{n} \backslash \mathbf{B}\right) \rightarrow \mathcal{C}_{\nu}^{0, \alpha}\left(\mathbb{R}^{n} \backslash \mathbf{B}\right)
$$

by

$$
\mathcal{F}(\varepsilon, w, \tau, u):=\mathcal{L}(|\varepsilon|, w, \tau, u)-f
$$


It is clear from Lemma 3.2 that $\mathcal{F}$ is of class $C^{2}$, since $n \geq 3$. Let $u_{0} \in \mathcal{C}_{\nu, \mathcal{D}}^{2, \alpha}\left(\mathbb{R}^{n} \backslash \mathbf{B}\right)$ be the unique solution to $\Delta_{0} u_{0}=f$. We have $\mathcal{F}\left(0,0,0, u_{0}\right)=0$ and by Theorem 2 , $\partial_{u} \mathcal{F}\left(0,0,0, u_{0}\right)$ is invertible. By the implicit function theorem, there exists a unique $u_{\varepsilon, w, \tau}$ satisfying $\mathcal{F}\left(\varepsilon, w, \tau, u_{\varepsilon, w, \tau}\right)=0$, with $u_{0,0,0}=u_{0}$. We then conclude that, provided $c_{0}$ and $\rho_{0}$ small, the linear map

$$
\mathcal{L}(\rho, w, \tau \cdot): \mathcal{C}_{\nu, \mathcal{D}}^{2, \alpha}\left(\mathbb{R}^{n} \backslash \mathbf{B}\right) \rightarrow \mathcal{C}_{\nu}^{0, \alpha}\left(\mathbb{R}^{n} \backslash \mathbf{B}\right)
$$

is invertible for every $\rho, w, \tau \in\left(0, \rho_{0}\right) \times \mathbf{B}_{c_{0}}(0) \times \mathbf{B}_{c_{0}}(0)$. The bound (3.16) also follows from the implicit function theorem.

\section{Approximate and Actual Solutions}

In this section we construct an approximate solution using the standard Greens function in Euclidean space and compare it to the solution of a corresponding Dirichlet problem.

For $w \in \mathbf{B}_{c_{0}}(0)$, we define

$$
v(x)=v_{\rho, w}(x)=\left|\left(1+w\left(\frac{x}{|x|}\right)\right) x\right|^{2-n} .
$$

We have the following expansion.

Lemma 4.1 For $\rho$ sufficiently small, the Laplacian of $v$ is given by

$$
\begin{aligned}
\Delta_{g_{\rho}} v= & \frac{(n-1)(n-2)^{2}}{2} \sigma \rho^{n-1} r^{1-2 n} \\
& -\frac{(n-1)(n-4)(n-2)^{2}}{2} \sigma \rho^{n-1} r^{1-2 n}\left\langle\frac{x}{r^{2}}, \tau\right\rangle+P_{2}(1 / \rho, x, w, \tau) .
\end{aligned}
$$

Proof By definition

$$
v(x)=|x|^{2-n}\left(1-(n-2) w+Q^{0}(w, \tau)\right),
$$

and so

$$
\begin{aligned}
\frac{\partial v}{\partial x_{i}} & =-(n-2) r^{-n}\left(1-(n-2) w+Q^{0}(w, \tau)\right) x_{i} \\
& -(n-2) r^{2-n} w_{i}+Q^{1}(w, \tau) \\
\partial_{i j}^{2} v & =-(n-2) r^{-n}(1-(n-2) w) \delta_{i j} \\
& +n(n-2) r^{-2-n}(1-(n-2) w) x_{i} x_{j}+(n-2)^{2} r^{-n} w_{j} x_{i} \\
& +(n-2)^{2} r^{-n} w_{i} x_{j}-(n-2) r^{2-n} w_{i j}+Q^{2}(w, \tau)
\end{aligned}
$$




$$
\Delta_{0}(v)=-(n-2) r^{2-n} \Delta_{0} w+Q^{2}(w, \tau)
$$

With this, we have

$$
\begin{aligned}
& \left(1-2 w-\sigma|z|^{1-n}\right) \Delta_{0}(v)=-(n-2) r^{2-n} \Delta_{0} w+Q^{2}(w, \tau) \\
& \quad\left[x_{i} w_{j}+x_{j} w_{i}+P_{1}(\rho, x, w, \tau)\right] \partial_{i j}^{2}(v)=P_{2}(\rho, x, w, \tau) . \\
& \quad-\left[3 w_{j}+x_{i} w_{i j}+\left[\Delta_{0} w\right] x_{j}+P_{2}(\rho, x, w, \tau]\right] \partial_{j}(v) \\
& =(n-2) r^{2-n} \Delta_{0} w+(n-2) r^{-n} x_{i} x_{j} w_{i j} \\
& \quad \frac{n-2}{2} \sigma \partial_{j}|y|^{1-n} \partial_{j}(v)=\frac{(n-1)(n-2)^{2}}{2} \sigma \rho^{1-n} r^{1-2 n} \\
& -\frac{(n-1)(n-4)(n-2)^{2}}{2} \sigma \rho^{1-n} r^{1-2 n}\left\langle\frac{x}{r^{2}}, \tau\right\rangle .
\end{aligned}
$$

However, $0=\partial_{i}\left(x^{j} w_{j}\right)=\delta_{i j} w_{j}+x^{j} w_{i j} \Rightarrow x^{i} x^{j} w_{i j}=-x^{i} \delta_{i j} j w_{j}=-x^{i} w_{i}=0$. The expansion in Lemma 4.1 now follows from Lemma 3.2 after replacing $\rho$ by $1 / \rho$.

Using Lemma 3.3, we construct a unique solution $\Psi_{\rho, w}(x)$ to the equation

$$
\begin{cases}\Delta_{g_{\rho}} \Psi_{\rho, \tau, w} & =-\Delta_{g_{\rho}} v \text { in } \mathbb{R}^{n} \backslash \mathbf{B} \\ \Psi_{\rho, w} & =1-v=(n-2) w+Q^{0}(w) \text { on } \partial \mathbf{B} .\end{cases}
$$

First choose $\chi \in \mathcal{C}_{c}^{\infty}\left(\mathbb{R}_{+}\right)$such that $\chi(t)=1$ for $1 / 2 \leq t \leq 1$, and define

$$
f(\rho, x, w)=-\Delta_{g_{\rho}}\left(\left((n-2) w+Q^{0}(w)\right) \chi(|x|)\right)-\Delta_{g_{\rho}} v \in \mathcal{C}_{v}^{0, \alpha}\left(\mathbb{R}^{n} \backslash \mathbf{B}\right)
$$

for any $v \in(2-n, 0)$. Next we use Lemma 3.3 to let $\widetilde{\Psi}_{\rho, \tau, w}$ be the unique solution of

$$
\Delta_{g_{\rho}} \widetilde{\Psi}_{\rho, \tau, w}(x)=f(\rho, x, w) \text { for } x \in \mathbb{R}^{n} \backslash \mathbf{B}, \quad \widetilde{\Psi}_{\rho, \tau, w}=0 \text { on } \partial \mathbf{B} .
$$

Observe that $f$ depends smoothly on $(\rho, w, \tau)$ and that the mapping $A \mapsto(A)^{-1}$ is smooth in the open set of linear invertible operator between two Banach spaces. We deduce that the mapping

$$
(\rho, \tau, w) \mapsto \widetilde{\Psi}_{\rho, \tau, w}
$$

is smooth. We then have

$$
\Psi_{\rho, \tau, w}=\widetilde{\Psi}_{\rho, \tau, w}+\left((n-2) w+Q^{0}(w)\right) \chi(|x|),
$$


which uniquely solves (4.7).

Finally we define

$$
\widehat{u}_{\rho, \tau, w}(x)=v(x)+\Psi_{\rho, \tau, w}(x),
$$

which satisfies

$$
\left\{\begin{array}{l}
\Delta_{g_{\rho}} \widehat{u}_{\rho, \tau, w}=0 \quad \text { in } \quad \mathbb{R}^{n} \backslash \mathbf{B} \\
\widehat{u}_{\rho, \tau, w}=1 \quad \text { on } \quad \partial \mathbf{B} .
\end{array}\right.
$$

Since $f(\rho, \cdot, w) \in \mathcal{C}_{v}^{0, \alpha}\left(\mathbb{R}^{n} \backslash \mathbf{B}\right),(4.8)$ and (3.16) imply that $\tilde{\Psi}_{\rho, w}(x) \rightarrow 0$ as $\quad|x| \rightarrow$ $\infty$. We conclude with (4.1), (4.9) and (4.10) that

$$
\widehat{u}_{\rho, \tau, w}(x) \rightarrow 0 \text { as }|x| \rightarrow \infty
$$

\section{Expansion of the Normal Derivative and Linear Analysis}

The aim of this section is to derive an expansion of the normal derivative of the solution $\widehat{u}_{\rho, \tau, w}$. We start by the computation of the interior unit normal vector to $\mathbf{B}$.

Lemma 5.1 Let $\Theta: \mathbb{R}^{n-1} \longrightarrow \boldsymbol{S}$ be a local parameterizition of $\boldsymbol{S}$ and set

$$
\Theta_{k}:=\partial_{k} \Theta \quad k=1, \ldots, n-1 .
$$

The interior unit normal vector field to $\mathbf{B}$ with respect to the metric $g_{\rho}$ is given by

$$
\begin{aligned}
\widehat{v}_{g_{\rho}}= & \frac{\widehat{\eta}}{\sqrt{\langle\hat{\eta}, \widehat{\eta}\rangle_{g_{\rho}}}}=\left[1-w-\frac{\sigma}{2} \rho^{1-n}(1-(n-1)\langle\Theta, \tau\rangle)+P_{1}(\rho, \Theta, w, \tau)\right] \\
& \times(-\Theta+\Upsilon),
\end{aligned}
$$

where

$$
\Upsilon=\sum_{m=1}^{n-1} a_{k} \Theta_{k} \quad \text { and } a_{k}=\left\langle\nabla_{S} w, \Theta_{k}\right\rangle+P_{1}(\rho, \Theta, w, \tau)
$$

Proof For each $\Theta \in \mathbf{S}$, the vectors

$$
\Theta_{\ell}:=\partial_{\ell} \Theta, \quad \ell=1, \ldots, n-1
$$

span the tangent space $T_{\Theta} \mathbf{S}$ and since $T_{\Theta} \mathbf{S} \simeq \mathbb{R}^{n-1}$, we may assume after orthonormalization that $\left\langle\Theta_{i}, \Theta_{j}\right\rangle=\delta_{i j}$. In addition having $\langle\Theta, \Theta\rangle=1$, it follows that $\left\langle\Theta, \Theta_{\ell}\right\rangle=0$ and $\left\langle\nabla_{\mathbf{S}} w, \Theta\right\rangle_{g_{\mathbf{S}}}=0$ on $\mathbf{S}$. With these indentities, Lemma 3.1 yields 


$$
\begin{aligned}
\left\langle\Theta_{i}, \Theta_{j}\right\rangle_{g_{\rho}} & =\left(1+2 w+\sigma|z|^{1-n}\right)\left\langle\Theta_{i}, \Theta_{j}\right\rangle+P_{1}(\rho, \Theta, w, \tau) \\
& =\left(1+2 w+\sigma|z|^{1-n}\right) \delta_{i j}+P_{1}(\rho, \Theta, w, \tau)
\end{aligned}
$$

and

$$
\left\langle\Theta, \Theta_{j}\right\rangle_{g_{\rho}}=P_{1}(\rho, \Theta, w, \tau)
$$

We look for a normal vector $\widehat{\eta}$ of $\mathbf{S}$ with respect to the metric $\widehat{g}$ in the form

$$
\widehat{\eta}=-\Theta+\Upsilon, \quad \Upsilon=\sum_{k=1}^{n-1} a_{k} \Theta_{k}
$$

The condition that $\widehat{\eta}$ is normal is thus equivalent to

$$
\left\langle\widehat{\eta}, \Theta_{\ell}\right\rangle_{g_{\rho}}=0, \quad \ell=1, \ldots, n-1 \Leftrightarrow\left\langle\Upsilon, \Theta_{\ell}\right\rangle_{g_{\rho}}=\left\langle\Theta, \Theta_{\ell}\right\rangle_{g_{\rho}}, \quad \ell=1, \ldots, n-1 .
$$

By Lemma 3.1,

$$
\begin{aligned}
\left\langle\Theta, \Theta_{\ell}\right\rangle_{g_{\rho}} & =\left(1+2 w+\sigma|z|^{1-n}\right)\left\langle\Theta, \Theta_{\ell}\right\rangle \\
& +\left[\left\langle\nabla_{\mathbf{S}} w, \Theta_{\ell}\right\rangle+\left\langle\nabla_{\mathbf{S}} w, \Theta\right\rangle_{g_{\mathbf{S}}}+P_{1}(\rho, \Theta, w, \tau)\right] \\
& =\left\langle\nabla_{\mathbf{S}} w, \Theta_{\ell}\right\rangle+P_{1}(\rho, \Theta, w, \tau) .
\end{aligned}
$$

We also have

$$
\left\langle\Upsilon, \Theta_{\ell}\right\rangle_{g_{\rho}}=\sum_{k=1}^{n-1} a_{k}\left\langle\Theta_{k}, \Theta_{\ell}\right\rangle_{g_{\rho}}
$$

Now using (5.6) and (5.8),

$$
\sum_{k=1}^{n-1} a_{k}\left\langle\Theta_{k}, \Theta_{\ell}\right\rangle_{g_{\rho}}=\left\langle\nabla_{\mathbf{S}} w, \Theta_{\ell}\right\rangle+P_{1}(\rho, \Theta, w, \tau), \quad \ell=1, \ldots, n-1
$$

Set

$$
\begin{aligned}
& b_{\ell}:=\left\langle\nabla_{\mathbf{S}} w, \Theta_{\ell}\right\rangle+P_{1}(\rho, \Theta, w, \tau) \\
& \widetilde{g}_{k \ell}:=\left\langle\Theta_{k}, \Theta_{\ell}\right\rangle_{g_{\rho}}=\left(1+2 w+\sigma|z|^{1-n}\right) \delta_{i j}+P_{1}(\rho, \Theta, w, \tau),
\end{aligned}
$$

where the last equality follows from (5.3). Then (5.8) reads,

$$
\sum_{k=1}^{n-1} a_{k} \widetilde{g}_{k \ell}=b_{\ell}, \quad \ell=1, \ldots, n-1
$$


and we deduce with (5.9) the coefficients

$$
a_{k}=\sum_{\ell=1}^{n-1} b_{\ell} \widetilde{g}^{k \ell}=\left\langle\nabla_{\mathbf{S}} w, \Theta_{k}\right\rangle+P_{1}(\rho, \Theta, w, \tau), \quad k=1, \ldots, n-1
$$

Next we compute

$$
\langle\widehat{\eta}, \widehat{\eta}\rangle_{g_{\rho}}=\langle\Theta, \Theta\rangle_{g_{\rho}}-2\langle\Theta, \Upsilon\rangle_{g_{\rho}}+\langle\Upsilon, \Upsilon\rangle_{g_{\rho}}
$$

We have by Lemma 3.1,

$$
\langle\Theta, \Theta\rangle_{g_{\rho}}=\left(1+2 w+\sigma|z|^{1-n}+P_{0}(\rho, \Theta, w, \tau)\right)
$$

and from (5.4),

$$
\langle\Theta, \Upsilon\rangle_{g_{\rho}}=\sum_{k=1}^{n-1} a_{k}\left\langle\Theta, \Theta_{\ell}\right\rangle_{g_{\rho}}=P_{1}(\rho, \Theta, w, \tau)
$$

In addition,

$\langle\Upsilon, \Upsilon\rangle_{g_{\rho}}=\sum_{k, \ell=1}^{n-1} a_{k} a_{\ell}\left\langle\Theta_{k}, \Theta_{\ell}\right\rangle_{g_{\rho}}=\sum_{\ell=1}^{n-1} a_{\ell}\left(\sum_{k=1}^{n-1} a_{\ell} \widetilde{g}^{k \ell}\right)=\sum_{\ell=1}^{n-1} a_{\ell} b_{\ell}=\sum_{k, \ell=1}^{n-1} b_{k} b_{\ell} \widetilde{g}^{k \ell}$

where we have used (5.11) to get the last equality. Using (5.10) and (5.9), it follows that

$$
\langle\Upsilon, \Upsilon\rangle_{g_{\rho}}=\sum_{\ell=1}^{n-1} b_{\ell}^{2}+P_{1}(\rho, \Theta, w, \tau)=P_{1}(\rho, \Theta, w, \tau)
$$

We gather (5.13), (5.14) and (5.15) to obtain

$$
\langle\widehat{\eta}, \widehat{\eta}\rangle_{g_{\rho}}=\left(1+2 w+\sigma|z|^{1-n}+P_{1}(\rho, \Theta, w, \tau)\right)
$$

The normal interior unit vector field to $\mathbf{B}$ for the metric $g_{\rho}$ is then given by

$$
\widehat{v}_{g_{\rho}}=\frac{\widehat{\eta}}{\sqrt{\langle\widehat{\eta}, \widehat{\eta}\rangle_{g_{\rho}}}}=\left(1-w-\frac{\sigma}{2}|z|^{1-n}+P_{1}(\rho, \Theta, w, \tau)\right)(-\Theta+\Upsilon),
$$

and (5.1) follows from (3.8). 


\subsection{Expansion of the Normal Derivative}

The following proposition yields the expansion of the normal derivative of $\widehat{u}_{\rho, \tau, w}$ with respect to the metric $g_{\rho}$.

Proposition 4 For $\rho$ sufficiently small the normal derivative of $\widehat{u}=\widehat{u}_{\rho, \tau, w}=v+$ $\Psi_{\rho, \tau w}$ with respect to the metric $g_{\rho}$ on $\partial \mathbf{B}$ is given by

$$
\frac{\partial \widehat{u}}{\partial \widehat{v}_{g_{\rho}}}=(n-2)\left(1-\frac{\sigma}{2} \rho^{n-1}\right)+H(\rho, \tau, w),
$$

where

$$
\begin{aligned}
& H(\rho, \tau, w)(x):=(n-1)(n-2)\left(\frac{\sigma}{2} \rho^{n-1}\langle x, \tau\rangle-w+P_{1}(1 / \rho, x, w, \tau)\right) \\
& +\widehat{v}_{g_{\rho}}^{i} \frac{\partial \Psi_{\rho, \tau, w}}{\partial x_{i}}
\end{aligned}
$$

and $\Psi_{\rho, \tau, w}$ is solution of (4.7).

Proof Recall our solution $\widehat{u}_{\rho, \tau, w}(x)=v(x)+\Psi_{\rho, \tau, w}(x)$, where $v$ is given by (4.1) and $\Psi_{\rho, \tau, w}$ satisfies (4.7).

We first compute $g_{\rho}\left(\nabla_{g_{\rho}} v, \widehat{v}_{g_{\rho}}\right)$. By Lemma 3.1 and (4.4)

$$
\begin{aligned}
\nabla_{g_{\rho}}^{j} v & =\sum_{i=1}^{n} g_{\rho}^{i j} \frac{\partial v}{\partial x_{i}}=\left[\left(1-2 w-\sigma|z|^{1-n}\right) \delta_{i j}-\left(x_{i} w_{j}+x_{j} w_{i}\right)+P_{0}(\rho, x, w, \tau)\right] \times \\
& {\left[-(n-2)\left(1-(n-2) w+Q^{0}(w)\right) x_{i}-(n-2) w_{i}+Q^{1}(w)\right] } \\
& =-(n-2)\left(\left(1-\sigma|z|^{1-n}\right) x_{j}-n w x_{j}+P_{1}(\rho, x, w, \tau)\right) \text { on } \partial \mathbf{B}
\end{aligned}
$$

which yields

$$
\begin{aligned}
g_{\rho}( & \left.-\Theta, \nabla_{g_{\rho}} v\right) \\
= & -\left[\left(1+2 w+\sigma|z|^{1-n}\right)\left\langle\Theta, \nabla_{g_{\rho}} v\right\rangle+\left\langle\nabla w, \nabla_{g_{\rho}} v\right\rangle\right. \\
& \left.+\langle\nabla w, \Theta\rangle\left\langle\Theta, \nabla_{g_{\rho}} v\right\rangle+P_{1}(\rho, \Theta, w, \tau)\right] \\
= & -\left[\left(1+2 w+\sigma|z|^{1-n}\right)\left\langle\Theta, \nabla_{g_{\rho}} v\right\rangle+P_{1}(\rho, \Theta, w, \tau)\right] .
\end{aligned}
$$

On the other hand,

$$
\left\langle\Theta, \nabla_{g_{\rho}} v\right\rangle=-(n-2)\left(1-\sigma|z|^{1-n}-n w+P_{1}(\rho, \Theta, w, \tau)\right),
$$


so we may rewrite our expression for $g_{\rho}\left(-\Theta, \nabla_{g_{\rho}} v\right)$ as

$$
g_{\rho}\left(-\Theta, \nabla_{g_{\rho}} v\right)=(n-2)\left(1+(2-n) w+P_{1}(\rho, \Theta, w, \tau)\right)
$$

Additionally,

$$
\begin{aligned}
& g_{\rho}\left(\Upsilon, \nabla_{g_{\rho}} v\right)=\sum_{k=1}^{n} a_{k} g_{\rho}\left(\Theta_{k}, \nabla_{g_{\rho}} v\right)=\sum_{k=1}^{n} a_{k} g_{\rho}\left(\Theta_{k}, \Theta\right) \\
& \quad+P_{1}(\rho, \Theta, w, \tau)=P_{1}(\rho, \Theta, w, \tau)
\end{aligned}
$$

where we have used (5.8) in the last equality. Finally, making use of (5.16), (5.20) and (5.19), we obtain

$$
\begin{aligned}
g_{\rho}\left(\nabla_{g_{\rho}} v, \widehat{v}_{g_{\rho}}\right)= & \left(1-w-\frac{\sigma}{2}|z|^{1-n}+P_{1}(\rho, \Theta, w, \tau)\right) g_{\rho}\left(-\Theta+\Upsilon, \nabla_{\widehat{g}} v\right) \\
= & (n-2)\left(1-w-\frac{\sigma}{2}|z|^{1-n}+P_{1}(\rho, \Theta, w, \tau)\right) \\
& \left(1+(2-n) w+P_{1}(\rho, \Theta, w)\right) \\
= & (n-2)\left(1-\frac{\sigma}{2} \rho^{1-n}+\frac{\sigma(n-1)}{2} \rho^{1-n}\langle x, \tau\rangle-(n-1) w\right. \\
& \left.+P_{1}(\rho, \Theta, w, \tau)\right)
\end{aligned}
$$

and

$$
g_{\rho}\left(\nabla_{g_{\rho}} \Psi_{\rho, \tau, w}, \widehat{v}_{g_{\rho}}\right)=g_{\rho}^{i j}\left(g_{\rho}\right)_{j k} \widehat{v}_{g_{\rho}}^{k} \frac{\partial \Psi_{\rho, \tau, w}}{\partial x_{i}}=\delta_{i k} \widehat{v}_{g_{\rho}}^{k} \frac{\partial \Psi_{\rho, \tau, w}}{\partial x_{i}}=\widehat{v}_{g_{\rho}}^{i} \frac{\partial \Psi_{\rho, \tau, w}}{\partial x_{i}}
$$

The expansion (5.17) then follows from (5.21) and (5.22) replacing $\rho$ by $1 / \rho$.

\subsection{Linear Analysis of the Normal Derivative}

In this section, we analyse (5.17) and (5.18) in detail and compute the operators that will allow us to solve

$$
\frac{\partial \widehat{u}}{\partial \widehat{v}_{g_{\rho}}}=\text { Const }
$$

Scanning (5.18), (4.7) and Lemma (4.1), we observe that

$$
H(0,0,0) \equiv 0
$$


With the condition (5.24), an obvious attempt is to consider the equation

$$
H(\rho, \tau, w)=0
$$

and apply the implicit function theorem to find $\tau$ and $w$ as functions of a small variable $\rho$. As shown in Proposition 5 below, the linearised operator of $H$ with respect to $w$ has a non trivial kernel. To solve (5.25), we then need to project on this kernel and first express $\tau$ in terms of $\rho$ and $w$. Still there are some challenges to overcome. Indeed, since $\tau$ appears with a factor of $\rho^{n-1}$ in (5.18) and (4.2), the derivative of $H$ with respect to $\tau$ evaluated at $(0,0,0)$ is zero. We compensate for this by multiplying the equation (5.25) by $\rho^{1-n}$ after replacing $w$ by $\rho^{n-1} w$, leading naturally to the function

$$
G(\rho, \tau, w):=\rho^{1-n} H\left(\rho, \tau, \rho^{n-1} w\right) .
$$

From (5.33) and (5.18),

$$
\begin{aligned}
G(\rho, \tau, w)(x) & =(n-1)(n-2)\left(\frac{\sigma}{2}\langle x, \tau\rangle-w+\rho^{1-n} P_{1}\left(1 / \rho, \Theta, \rho^{n-1} w, \tau\right)\right) \\
& +\widehat{v}_{g_{\rho}}^{i}\left(\rho^{n-1} w\right) \frac{\partial \bar{\Psi}_{\rho, \tau, w}}{\partial x_{i}}
\end{aligned}
$$

where

$$
\bar{\Psi}_{\rho, \tau, w}:=\rho^{1-n} \Psi_{\rho, \tau, \rho^{n-1} w} .
$$

We emphasise that the function $\Psi_{\rho, \tau, \rho^{n-1} w}$ is the unique solution of (4.7) with $w$ replaced by $\rho^{n-1} w$ in both the metric coefficients of $g_{\rho}$ involved in the operator $\Delta_{g_{\rho}}$, and the right hand side of the interior and boundary equations. We keep the notation $\Delta_{g_{\rho}}$ for this new Laplacian. The quantity $\widehat{v}_{g_{\rho}}^{i}\left(\rho^{n-1} w\right)$ is the ith-component of the unit vector $\widehat{v}_{g_{\rho}}$ in (5.17) where $w$ is also replaced with $\rho^{n-1} w$.

By Lemma 4.1 that $\bar{\Psi}_{\rho, \tau, w}$ is solution the unique solution of

$$
\left\{\begin{aligned}
\Delta_{g_{\rho}} \bar{\Psi}_{\rho, \tau, w} & =-\frac{(n-1)(n-2)^{2}}{2} \sigma r^{1-2 n}+\frac{(n-1)(n-4)(n-2)^{2}}{2} \sigma r^{1-2 n}\left\langle\frac{x}{r^{2}}, \tau\right\rangle \\
& +\rho^{1-n} P_{2}\left(1 / \rho, x, \rho^{n-1} w, \tau\right) \text { in } \mathbb{R}^{n} \backslash \mathbf{B} \\
\bar{\Psi}_{\rho, \tau, w} & =(n-2) w+\rho^{1-n} Q^{0}\left(\rho^{n-1} w, \tau\right) \text { on } \partial \mathbf{B} .
\end{aligned}\right.
$$

In addition,

$$
\bar{\Psi}_{0,0,0}=C_{1}(n, \sigma) \mathcal{K}
$$

where

$$
C_{1}(n, \sigma)=-\frac{(n-1)(n-2)^{2}}{2} \sigma
$$


and $\mathcal{K}$ is the unique solution of

$$
\begin{cases}\Delta \mathcal{K} & =r^{1-2 n} \quad \text { in } \quad \mathbb{R}^{n} \backslash \mathbf{B} \\ \mathcal{K} & =0 \quad \text { on } \quad \partial \mathbf{B} .\end{cases}
$$

It is plain that the function $\mathcal{K}$ is radial, and one obtain by Gauss-Green formula $\partial_{\nu} \mathcal{K}=$ $-\mathcal{K}^{\prime}(1)=\frac{1}{n-1}$.

Now from (5.29) and (5.27) it follows that

$$
G(0,0,0)=C_{1}(n, \sigma) \partial_{\nu} \mathcal{K}=\frac{C_{1}(n, \sigma)}{n-1}=-\frac{(n-2)^{2}}{2} \sigma .
$$

The non linear problem under consideration is then to show that provided $\rho$ is small, we can find $\tau$ and $w$ such that

$$
G(\rho, \tau, w)+\frac{(n-2)^{2}}{2} \sigma=0 .
$$

We start by studying the operator $G$.

Proposition 5 Let

$$
G(\rho, \tau, w):=\rho^{1-n} H\left(\rho, \tau, \rho^{n-1} w\right) .
$$

Then

$$
\left[\left.D_{\tau}\right|_{(\rho, \tau, w)=(0,0,0)} G(\rho, \tau, w)\right] \cdot \tau=\frac{(n-1)(n-2)}{2}\left(1+\frac{(n-2)(n-4)}{2 n-1}\right) \sigma\langle x, \tau\rangle
$$

and

$$
\left[\left.D_{w}\right|_{(\rho, \tau, w)=(0,0,0)} G(\rho, \tau, w)\right] \cdot w=(n-2)\left(\partial_{\nu} \psi_{w}-(n-1) w\right)
$$

where $v=-x$ and $\psi_{w}$ is the unique solution to

$$
\begin{cases}\Delta_{0} \psi_{w} & =0 \text { in } \quad \mathbb{R}^{n} \backslash \mathbf{B} \\ \psi_{w} & =w \text { on } \quad \partial \mathbf{B}\end{cases}
$$

Proof We first prove (5.35). Set

$$
\mathcal{U}:=\left\{w \in C^{2, \alpha}(\partial \mathbf{B}): w>0\right\}
$$

and consider the smooth map $T: \mathbb{R}_{+}^{*} \times \mathbb{R}_{+}^{*} \times \mathcal{U} \rightarrow C^{0, \alpha}(\partial \mathbf{B})$ defined by

$$
T(\rho, \tau, w):=\Delta_{g_{\rho}} \bar{\Psi}_{\rho, \tau, w}=g_{\rho}^{i j} \partial_{i j} \bar{\Psi}_{\rho, \tau, w}+\frac{1}{\sqrt{\left|g_{\rho}\right|}} \partial_{i}\left(\sqrt{\left|g_{\rho}\right|} g_{\rho}^{i j}\right) \partial_{j} \bar{\Psi}_{\rho, \tau, w}
$$


For every $\omega \in C^{2, \alpha}(\partial \mathbf{B})$ we have

$$
\left[D_{w} T(\rho, \tau, w)\right] \omega=\Delta_{g_{\rho}}\left[D_{w} \bar{\Psi}_{\rho, \tau, w}\right] \omega+h_{w}^{i j} \partial_{i j} \bar{\Psi}_{\rho, \tau, w}+\ell_{w}^{j} \partial_{j} \bar{\Psi}_{\rho, \tau, w}
$$

with

$$
h_{\omega}^{i j}:=\left[D_{w} g_{\rho}^{i j}\right] \omega \quad \text { and } \quad \ell_{\omega}^{j}:=\left[D_{w} \frac{1}{\sqrt{\left|g_{\rho}\right|}} \partial_{i}\left(\sqrt{\left|g_{\rho}\right|} g_{\rho}^{i j}\right)\right] \omega .
$$

As already explained before (5.28), $w$ is replaced by $\rho^{n-1} w$ in the metric coefficients of $g_{\rho}$ involved in $\Delta_{g_{\rho}}$. Hence evaluating $(5.37)$ at $(\rho, \tau, w)=(0,0,0)$ and using (3.12) and Lemmas 3.1-3.2 we find after differentiating the right hand of (5.28) that the function

$$
\psi_{w}:=\frac{1}{n-2}\left[\left.D_{w}\right|_{(\rho, \tau, w)=(0,0,0)} \bar{\Psi}_{\rho, \tau, w}\right] \cdot w
$$

satisfies

$$
\left\{\begin{array}{l}
\Delta \psi_{w}=0 \quad \text { in } \quad \mathbb{R}^{n} \backslash \mathbf{B} \\
\psi_{w}=w \quad \text { on } \partial \mathbf{B}
\end{array}\right.
$$

We also have from (5.1),

$$
\left[\left.D_{w}\right|_{(\rho, \tau, w)=(0,0,0)} \widehat{v}_{g_{\rho}}^{i}\left(\rho^{n-1} w\right)\right] \cdot w=0=\left[\left.D_{\tau}\right|_{(\rho, \tau, w)=(0,0,0)} \widehat{v}_{g_{\rho}}^{i}\left(\rho^{n-1} w\right)\right] \cdot \tau .
$$

Indead, $\widehat{v}_{g_{\rho}}^{i}\left(\rho^{n-1} w\right)$ is the ith-component of the unit vector $\widehat{v}_{g_{\rho}}$ in Lemma (5.1) with $\rho^{n-1} w$ in place of $w$ and $\rho$ replace by $1 / \rho$. This together with the definition of the functions $P_{i}$ in (3.4) allows us to recover (5.40). Now returning to (5.27) and using (5.38), (5.39) and (5.40) we get

$$
\left[\left.D_{w}\right|_{(\rho, \tau, w)=(0,0,0)} G(\rho, \tau, w)\right] \cdot w=(n-2)\left(\partial_{\nu} \psi_{w}-(n-1) w\right),
$$

which is the desired expression in (5.35).

Next we prove (5.34). We use a similar argument as above and differentiate (5.28) with respect to $\tau$ at $(\rho, \tau, w)=(0,0,0)$. This yields a function

$$
F_{\tau}:=\left[\left.D_{\tau}\right|_{(\rho, \tau, w)=(0,0,0)} \bar{\Psi}_{\rho, \tau, w}\right] \cdot \tau
$$

satisfying

$$
\left\{\begin{array}{lc}
\Delta F_{\tau}=C(n, \sigma) r^{1-2 n}\left\langle\frac{x}{r^{2}}, \tau\right\rangle & \text { in } \quad \mathbb{R}^{n} \backslash \mathbf{B} \\
F_{\tau}=0 & \text { on } \partial \mathbf{B},
\end{array}\right.
$$


where

$$
C(n, \sigma):=\frac{(n-1)(n-4)(n-2)^{2}}{2} \sigma .
$$

This combined with (5.27) and (5.40) yields

$$
\left[\left.D_{\tau}\right|_{(\rho, \tau, w)=(0,0,0)} G(\rho, \tau, w)\right] \cdot \tau=\frac{(n-1)(n-2)}{2} \sigma\langle x, \tau\rangle+\partial_{\nu} F_{\tau} .
$$

Next, we show that

$$
\partial_{\nu} F_{\tau}=\frac{C(n, \sigma)}{2 n-1}\langle\cdot, \tau\rangle \quad \text { on } \mathbf{S}, \quad \text { with } \quad v=-x
$$

Indeed, let

$$
\Pi: L^{2}(\mathbf{S}) \rightarrow L^{2}(\mathbf{S})
$$

be the orthogonal projection on $\operatorname{span}\left\{x_{1} ; \ldots ; x_{n}\right\}$ and consider the function

$$
X^{i}(x):=\mathbb{K}\left(x^{i}\right)=|x|^{2-n} \frac{x^{i}}{|x|^{2}}
$$

We know that $\Delta_{0} X^{i}=0$ in $\mathbb{R}^{n} \backslash\{0\}$. We multiply (5.41) with $X^{i}$ and integrate by parts to get

$$
\begin{aligned}
\int_{\mathbf{S}} \partial_{\nu} F_{\tau}(x) x^{i} \mathrm{~d} \mu_{S} & =C(n, \sigma) \int_{\mathbb{R}^{n} \backslash \mathbf{B}}|x|^{-1-n}|x|^{2-n}\left\langle\frac{x}{|x|^{2}}, \tau\right\rangle X^{i}(x) \mathrm{d} \mu \\
& =C(n, \sigma) \tau^{i} \int_{\mathbf{S}}\left(x^{i}\right)^{2} d \mu \int_{1}^{\infty} r^{1-3 n} r^{n-1} d r .
\end{aligned}
$$

This implies that

$$
\int_{\mathbf{S}} \partial_{\nu} F_{\tau}(x) x^{i} d \mu_{S}=\frac{C(n, \sigma)|\partial \mathbf{B}|}{2 n-1} \tau^{i}=\frac{C(n, \sigma)}{2 n-1} \int_{\mathbf{S}}\langle x, \tau\rangle x^{i} \mathrm{~d} \mu_{S} .
$$

From this, we deduce that

$$
\Pi \partial_{\nu} F_{\tau}(x)=\frac{C(n, \sigma)\langle\tau, x\rangle}{2 n-1} \quad \text { for } x \in \mathbf{S} .
$$

We claim that

$$
\Pi^{\perp} \partial_{\nu} F_{\tau}=0 .
$$


To see this, we let $Y_{i}^{k}$ be the spherical harmonics for which $Y_{i}^{1}=x^{i}$ for $i=1, \ldots, n$, corresponding to the eigenvalues $k(k+n-2)$ on sphere. We suppose that $k \neq 1$. We then define

$$
X_{i}^{k}(x)=\mathbb{K}\left(Y_{i}^{k}\right)(x)
$$

Then $X_{i}^{k}$ are admissible test functions in (5.41). We observe that the right hand side in (5.41) is in $L^{2}\left(\mathbb{R}^{n} \backslash \mathbf{B}\right)$. Therefore by simple arguments, we have that $F_{\tau} \in H^{1}\left(\mathbb{R}^{n} \backslash \mathbf{B}\right)$. Using the decomposition in spherical harmonics of $F_{\tau}$, we can see that $F_{\tau}(x)=$ $f(|x|)\langle x, \tau\rangle$, for some some function $f$. From this we can multiply (5.41) by $X_{i}^{k}$ and use the Gauss-Green formula to deduce that

$$
\int_{\mathbf{S}} \partial_{\nu} F_{\tau}(x) Y_{i}^{k}(x) \mathrm{d} \mu_{S}=\tau^{i} \int_{\mathbb{R}^{n} \backslash \mathbf{B}}|x|^{-n}|x|^{-n} x^{i} X_{i}^{k}(x) \mathrm{d} \mu=0, \quad k \neq 1,
$$

as claimed. Gathering (5.42), (5.43) and (5.44), we obtain (5.34).

\subsection{The Spectral Properties of the Operator $\mathbb{L}$}

We study the spectral properties of the operator $\mathbb{L}$ in (5.35) defined by

$$
\mathbb{L}(w):=\partial_{\nu} \psi_{w}-(n-1) w
$$

where $\psi_{w}$ satisfies (5.36). We consider the Kelvin transform (see (3.14) and (3.15), and observe $\mathbb{K}^{-1}=\mathbb{K}$ ) of $\psi_{w}$

$$
\mathbb{K}\left(\psi_{w}\right)(x)=|x|^{2-n} \psi_{w}\left(\frac{x}{|x|^{2}}\right)
$$

which satisfies

$$
\Delta\left(\mathbb{K}\left(\psi_{w}\right)\right)=0 \quad \text { in } \mathbf{B} \backslash\{0\} .
$$

Moreover by direct computations, we have

$$
\nabla\left(\mathbb{K}\left(\psi_{w}\right)\right) \cdot x=-\nabla \psi_{w} \cdot x+(2-n) w=\partial_{\nu} \psi_{w}+(2-n) w \quad \text { in } \partial \mathbf{B} .(5.48)
$$

Since $\psi_{w} \in \mathcal{C}_{v}^{2, \alpha}\left(\mathbb{R}^{n} \backslash \mathbf{B}\right)$ with $v \in(2-n, 0)$, we see that

$$
\lim _{|x| \rightarrow \infty}|x|^{2-n} \psi_{w}(x)=0 .
$$

Therefore

$$
\lim _{|x| \rightarrow 0}|x|^{n-2} \mathbb{K}\left(\psi_{w}\right)(x)=0
$$


so the origin is a removable singularity for $\mathbb{K}\left(\psi_{w}\right)$ and thus $\mathbb{K}\left(\psi_{w}\right)$ solves

$$
\left\{\begin{array}{l}
\Delta \mathbb{K}\left(\psi_{w}\right)=0 \quad \text { in } \quad \mathbf{B} \\
\mathbb{K}\left(\psi_{w}\right)=w \quad \text { on } \quad \partial \mathbf{B} .
\end{array}\right.
$$

By elliptic regularity theory, $\mathbb{K}\left(\psi_{w}\right) \in \mathcal{C}^{2, \alpha}(\overline{\mathbf{B}})$. From (5.48) and the definition of $\mathbb{L}$ we get

$$
w \mapsto \mathbb{L}(w)=\partial_{\tilde{v}}\left(\mathbb{K}\left(\psi_{w}\right)\right)-w
$$

where $\tilde{v}=\Theta$.

Thanks to [10], the spectrum of the operator $\mathbb{L}$ is given by

$$
\lambda_{k}=k-1, \quad k \in \mathbb{N}
$$

meaning that the kernel of the operator $\mathbb{L}$ is given by the space $V_{1}$ spanned by linear coordinates on the sphere $\mathbf{S}$

$$
V_{1}:=\left\{\Theta^{i}, i=1, \ldots, n\right\}
$$

Moreover there exists a constant $C>0$ such that

$$
\|w\|_{C^{2, \alpha}(\mathbf{S})} \leq C\|\mathbb{L}(w)\|_{C^{1, \alpha}(\mathbf{S})}
$$

provided $w \in \Pi^{\perp} C^{, \alpha}(\mathbf{S})$.

\section{Solving the Nonlinear Problem}

We want to show that provided $\rho$ is small, we can find $\tau$ and $w$ such that (5.32) holds. That is

$$
G(\rho, \tau, w)+\frac{(n-2)^{2}}{2} \sigma=0 .
$$

Define

$$
\widetilde{G}(\rho, \tau, w):=G(\rho, \tau, w)+\frac{(n-2)^{2}}{2} \sigma .
$$

Then from (5.31),

$$
\widetilde{G}(0,0,0)=0
$$


We denote by $\Pi$ the $L^{2}$-orthogonal projection from $\mathcal{C}^{1, \alpha}(\mathbf{S})$ onto $V_{1}$ and $T: V_{1} \rightarrow \mathbb{R}^{n}$ the isomorphism sending $\left.x_{i}\right|_{\partial \mathbf{B}}$ to $e_{i}$. We also define $\widetilde{\Pi}:=T \circ \Pi$,

$$
\widetilde{K}:=\widetilde{\Pi} \circ \widetilde{G}:\left(0, \delta_{0}\right) \times \mathbf{B}_{c_{0}}(0) \times \mathbf{B}_{c_{0}}(0) \longrightarrow \mathbb{R}^{n}
$$

and consider the equation

$$
\widetilde{K}(\rho, \tau, w)=0
$$

The mapping $\widetilde{K}$ has the following properties:

- $\widetilde{K}(0,0,0)=0$. This is from (6.2),

- $\left.D_{\tau}\right|_{(\rho, \tau, w)=(0,0,0)} \widetilde{K}$ is a the identity in $\mathbb{R}^{n}$ times a constant, which follows from (5.34).

Applying the implicit function theorem, we find a unique smooth mapping

$$
\left(-r_{0}, r_{0}\right) \times \mathbf{B}_{k_{1}}(0) \longrightarrow \mathbf{B}_{k_{2}}(0) \subset \mathbb{R}^{n}, \quad(\rho, w) \mapsto \tau(\rho, w)
$$

defined for some constants $r_{0}, k_{1}$ and $k_{2}$ in $\mathbb{R}_{+}^{*}$ such that

$$
\widetilde{K}(\rho, \tau(\rho, w), w)=0 \quad \text { for all }(\rho, w) \in\left(-r_{0}, r_{0}\right) \times \mathbf{B}_{k_{0}}(0)
$$

Net we provide estimates for the function $\tau(\rho, w)$ in (6.4). Observe that since $\widetilde{\Pi} \circ \mathbb{L}=$ $0,(6.4)$ is equivalent to

$$
C^{\prime}(n, \sigma) \tau+\widetilde{\Pi}\left[\rho^{1-n} P_{1}\left(1 / \rho, x, \rho^{n-1} w, \tau\right)\right]=0 \quad \text { on } \quad \mathbf{S}
$$

where $C^{\prime}(n, \sigma)$ is the constant appearing in (5.34). This can be seen by writing the Taylor expansion of $G$ using (5.34) and (5.35). We then deduce from (3.3) and (3.4) the estimates

$$
|\tau(\rho, w)| \leq C \rho \quad \text { and } \quad\left|D_{w} \tau(\rho, w)\right| \leq C \rho^{n-1}
$$

Now replace $\tau$ by $\tau(\rho, w)$ in (6.1) and consider the equation

$$
\Pi^{\perp}(\widetilde{G}(\rho, \tau, w))=0 .
$$

From (5.35) and the estimates in (6.6), the mapping

$$
\left.D_{w}\right|_{(\rho, \tau, w)=(0,0,0)} \Pi^{\perp} \circ \widetilde{G}=\mathbb{L}: V_{1}^{\perp} \longrightarrow \mathbb{L}\left(V_{1}^{\perp}\right),
$$

is an isomorphism from Sect. 5.3. Hence, there exists a unique solution $w(\rho)$ to (6.7) for small $\rho \in\left(0, R_{0}\right)$. Using (5.34) and (5.35),

$$
\mathbb{L}(w(\rho))+\Pi^{\perp}\left[\rho^{1-n} P_{1}\left(1 / \rho, x, \rho^{n-1} w(\rho), \tau(\rho, w)\right)\right]=0 \quad \text { on } \quad \mathbf{S},
$$


and from (5.51) and (3.4),

$$
\|w(\rho)\|_{C^{2, \alpha}(\mathbf{S})} \leq C \rho .
$$

Decreasing $R_{0}$ if necessary, the analysis of the previous section establishes the first statement of Theorem 1 with $\rho_{0}=\frac{1}{R_{0}}$ and

$$
\Omega_{\rho}=\widetilde{\Omega_{\frac{1}{\rho}}}, \quad \rho \in\left(\rho_{0},+\infty\right)
$$

where

$$
\widetilde{\Omega_{\rho}}:=\Phi_{\frac{1}{\rho}, \tau(\rho, w(\rho)), \rho^{n-1} w(\rho)}\left(\mathbb{R}^{n} \backslash \mathbf{B}\right), \quad \rho \in\left(0, R_{0}\right) .
$$

In addition combining (5.17), (5.18), (5.26) (5.27) and (6.1), we have for every $\rho \in$ $\left(0, R_{0}\right)$

$$
H\left(\rho, \tau(\rho, w(\rho)), \rho^{n-1} w(\rho)\right)=-\frac{(n-2)^{2}}{2} \sigma \rho^{n-1}
$$

and

$$
\frac{\partial \widehat{u}}{\partial \widehat{v}_{g_{\rho}}}=(n-2)\left(1-\frac{(n-1)}{2} \sigma \rho^{n-1}\right) .
$$

Recalling also (3.11), $\widehat{v}_{\widehat{g}}=\rho \widehat{v}_{g_{\rho}}$ and $\widehat{g}\left(\nabla_{\widehat{g}} \widehat{u}, \widehat{v}_{\widehat{g}}\right)=\rho g_{\rho}\left(\nabla_{g_{\rho}} \widehat{u}, \widehat{v}_{g_{\rho}}\right)$ we for every $\rho \in\left(0, R_{0}\right)$, Therefore, the constant $C(\rho, \sigma, n)$ in Theorem 1 is given by

$$
C(\rho, \sigma, n)=\frac{n-2}{\rho}\left(1-\frac{(n-1)}{2 \rho^{n-1}} \sigma\right), \quad \rho>\rho_{0} .
$$

It remains to show that the family $\left(\partial \Omega_{\rho}\right)_{\rho \in\left(\rho_{0},+\infty\right)}$ constitutes a smooth foliation.

\section{Foliation by Boundaries of Critical Capacitors}

Proposition 6 There exists a constant $\rho_{0}>1$ such that the family $\left(\partial K_{\rho}\right)_{\rho>\rho_{0}}$ constitutes a smooth foliation.

Proof We are proving that the family $\left(\partial \Omega_{\rho}\right)_{\rho \in\left(\rho_{0},+\infty\right)}$ constitutes a foliation of $M \backslash \Omega_{\rho_{0}}$. The proof is inspired by the argument in [3, Sect. 5] and [14, Pages 9-10].

Notice that $\partial \widetilde{\Omega_{\rho}}$ is given by

$$
\partial \widetilde{\Omega_{\rho}}=\Phi\left(\widetilde{S}_{\frac{1}{\rho}, \tau(\rho, w(\rho)), \rho^{n-1} w(\rho)}\right)
$$


where

$$
\widetilde{S}_{\frac{1}{\rho}, \tau, w}=\left\{y=\frac{1}{\rho}(x+\tau+w(x) x), \quad x \in \mathbf{S}\right\} .
$$

We define the functions

$$
h(\rho, x):=\frac{1}{\rho}\left(x+\tau(\rho, w(\rho))+\rho^{n-1} w_{\rho}(x) x\right) \text { and } v(\rho, x):=\frac{h(\rho, x)}{|h(\rho, x)|_{g_{\rho}}}, \quad x \in \mathbf{S} .
$$

By the estimates in (6.6) and (6.8), the function $v(\rho, \cdot)$ extends continuously at $\rho=0$ with $v(0, \cdot)=I_{\mathbf{S}}$ and for all $\rho$ small $v(\rho, \cdot)$ is a diffeomorphism from $\mathbf{S}$ into itself.

Thus for all $y \in \mathbf{S}$,

$$
h\left(\rho, v^{-1}(\rho, y)\right)=\left|h\left(\rho, v^{-1}(\rho, y)\right)\right|_{g_{\rho}} y .
$$

We put

$$
\tilde{\varphi}(\rho, y):=\left|h\left(\rho, v^{-1}(\rho, y)\right)\right|_{g_{\rho}},
$$

where $v^{-1}(\rho, \cdot)$ denotes the inverse of $v(\rho, \cdot)$. Then

$$
\widetilde{S}_{\frac{1}{\rho}, \tau(\rho, w(\rho)), \rho^{n-1} w(\rho)}=\widetilde{S}_{\rho}:=\{\tilde{\varphi}(\rho, y) y, \quad y \in \mathbf{S}\} .
$$

Using the estimates in (6.6) and (6.8) once again, we find

$$
\begin{aligned}
D_{x} h= & \frac{1}{\rho}\left(I_{\mathbf{S}}+\rho^{n-1} L^{1}\left(w_{\rho}\right) x+\rho^{n-1} w_{\rho}(x) I_{\mathbf{S}}\right)=\frac{1}{\rho}\left(I_{\mathbf{S}}+O(\rho)\right) \\
\frac{\partial h}{\partial \rho}= & -\frac{1}{\rho^{2}}\left(x+\tau-\rho \frac{\partial \tau}{\partial \rho}\right. \\
& \left.-\rho D_{w} \tau(\rho, w(\rho)) w_{\rho}^{\prime}+\rho^{n} w_{\rho}^{\prime}(x) x-(n-2) \rho^{n-1} w_{\rho}(x) x\right) \\
= & -\frac{1}{\rho^{2}}(x+O(\rho)) .
\end{aligned}
$$

Also, since

$$
\left|v^{-1}(\rho, y)\right|_{g_{\rho}}=1 \text { for all } \rho \in\left(0, R_{0}\right) \text { and } y \in \mathbf{S} \text {, }
$$

we have

$$
\left\langle v^{-1}(\rho, y), \partial_{\rho} v^{-1}(\rho, y)\right\rangle_{g_{\rho}}=0 \text { for all } \rho \in\left(0, R_{0}\right) \text { and } y \in \mathbf{S}
$$


Using this, we then obtain

$$
\begin{aligned}
\frac{\partial \tilde{\varphi}}{\partial \rho} & =\frac{1}{\left|h\left(\rho, v^{-1}(\rho, y)\right)\right|_{g_{\rho}}}\left\langle h\left(\rho, v^{-1}(\rho, y)\right), \frac{\partial h}{\partial \rho}\left(\rho, v^{-1}(\rho, y)\right)+D_{x} h\left(\partial_{\rho} v^{-1}\right)\right\rangle_{g_{\rho}} \\
& =\frac{1}{\left|v^{-1}(\rho, y)+O(\rho)\right|_{g_{\rho}}}\left\langle v^{-1}(\rho, y)+O(\rho),-\frac{1}{\rho} v^{-1}(\rho, y)+\partial_{\rho} v^{-1}+O(\rho)\right\rangle_{g_{\rho}} \\
& =\frac{1}{\rho\left|v^{-1}(\rho, y)+O(\rho)\right|_{g_{\rho}}}\left\langle v^{-1}(\rho, y)+O(\rho),-v^{-1}(\rho, y)+\rho \partial_{\rho} v^{-1}+O\left(\rho^{2}\right)\right\rangle_{g_{\rho}} \\
& =-\frac{1}{\rho}(1+O(\rho)) .
\end{aligned}
$$

We conclude the the function $\tilde{\varphi}(\rho, x)$ is strictly decreasing with respect to $\rho$ for $\rho$ small or equivalently $\tilde{\varphi}\left(\rho^{-1}, x\right)$ is strictly increasing for $\rho$ large. Thanks to (7.2), the family $\left(\widetilde{S}_{\frac{1}{\rho}}\right)_{\rho>\rho_{0}}$ constitutes a foliation of $\mathbb{R}^{n} \backslash \mathbf{B}_{\rho_{0}}$. Since $\Phi$ is a diffeomorphism and $\partial \Omega_{\rho}=\Phi^{\rho}\left(\widetilde{S}_{\frac{1}{\rho}}\right)$, we deduce that the family $\left(\partial \Omega_{\rho}\right)_{\rho \in\left(\rho_{0},+\infty\right)}$ foliates $M \backslash \Omega_{\rho_{0}}$ and the proof of Theorem 1 is complete.

Acknowledgements M. M. F. is partially supported by Alexander van Humboldt Foundation, J. R. was partially supported by the National Research Foundation of South Africa, and I. A. M. was partially supported by the Abbas Bahri Excellence Fellowship. We completed part of this research when M. M. F. visited the University of Cape Town, when J. R. visited the African Institute for Mathematical Sciences in Mbour, Senegal, and when I. A. M. visited the Department of Mathematics at the University of Rutgers. We thank all these institutions for their hospitality.

Funding Open Access funding enabled and organized by Projekt DEAL.

Open Access This article is licensed under a Creative Commons Attribution 4.0 International License, which permits use, sharing, adaptation, distribution and reproduction in any medium or format, as long as you give appropriate credit to the original author(s) and the source, provide a link to the Creative Commons licence, and indicate if changes were made. The images or other third party material in this article are included in the article's Creative Commons licence, unless indicated otherwise in a credit line to the material. If material is not included in the article's Creative Commons licence and your intended use is not permitted by statutory regulation or exceeds the permitted use, you will need to obtain permission directly from the copyright holder. To view a copy of this licence, visit http://creativecommons.org/licenses/by/4.0/.

\section{Appendix A: Variational Setting}

In this section we define two scale-invariant energies associated with capacity, and compute their first variations. We perform the computations below in Euclidean space, but again it is an easy exercise to carry them out in a Riemannian manifold with one asymptotically flat end.

We earlier defined the capacity function Cap on compact sets $K \subset \mathbb{R}^{n}$ in (1.1). A change of variables shows us the scaling law

$$
\operatorname{Cap}(R K)=R^{n-2} \operatorname{Cap}(K)
$$


for any $R>0$, and a quick compation gives $\operatorname{Cap}\left(\overline{\mathbf{B}}_{R}\right)=R^{n-2}$, with the equilibrium potention function $U(x)=R^{n-2}|x|^{2-n}$. As we discussed in the introduction, one can either normalize using volume or surface area, leading to the following two scaleinvariant functionals:

$$
\mathcal{E}_{0}(K)=\frac{\operatorname{Cap}(K)}{|K|^{\frac{n-2}{n}}}, \quad \mathcal{E}_{1}(K)=\frac{\operatorname{Cap}(K)}{|\Sigma|^{\frac{n-2}{n-1}}} .
$$

By (A.1) both $\mathcal{E}_{0}$ and $\mathcal{E}_{1}$ are scale-invariant.

To compute the first variation of both $\mathcal{E}_{0}$ and $\mathcal{E}_{1}$ we let $X:(-\varepsilon, \varepsilon) \times \mathbb{R}^{n} \rightarrow \mathbb{R}^{n}$ be a vector field, and let $\xi$ be its flow, defined by

$$
\xi:(-\varepsilon, \varepsilon) \times \mathbb{R}^{n} \rightarrow \mathbb{R}^{n}, \quad \frac{\partial \xi}{\partial t}(t, x)=X(t, x), \quad \xi(0, x)=x .
$$

Let $K_{t}=\xi(t, \cdot)(K)$ and let $U_{t}$ be the solution to (1.2) in $\Omega_{t}=\mathbb{R}^{n} \backslash K_{t}$. It will also be convenient to denote $\Sigma=\partial K=\partial \Omega$, and $\Sigma_{t}=\partial K_{t}=\partial \Omega_{t}$.

Lemma A.1 We have

$$
\begin{aligned}
& \left.\frac{\mathrm{d}}{\mathrm{d} t} \mathcal{E}_{0}\left(K_{t}\right)\right|_{t=0}=\frac{1}{n(n-2) \omega_{n}|K|^{\frac{n-2}{n}}}\left[\frac{(n-2)^{2} \omega_{n} \operatorname{Cap}(K)}{|K|} \int_{\Sigma}\langle X, \eta\rangle \mathrm{d} \sigma\right. \\
& \left.\quad-\int_{\Sigma}\langle X, \eta\rangle\left(\frac{\partial U}{\partial \eta}\right)^{2} \mathrm{~d} \sigma\right],
\end{aligned}
$$

where $\eta$ is the unit normal vector pointing into $K$.

Proof Differentiating the boundary condition $\left.U_{t}\right|_{\Sigma_{t}}=0$, we see

$$
\frac{\partial U}{\partial t}=-\langle X, \eta\rangle \frac{\partial U}{\partial \eta} \text { on } \Sigma_{t}
$$

Thus

$$
\begin{aligned}
\left.\frac{\mathrm{d}}{\mathrm{d} t}\right|_{t=0} \mathcal{E}_{0}\left(K_{t}\right)= & \left.\frac{1}{n(n-2) \omega_{n}} \frac{\mathrm{d}}{\mathrm{d} t}\right|_{t=0}|K|^{\frac{2-n}{n}} \int_{\Omega_{t}}\left|\nabla U_{t}\right|^{2} \mathrm{~d} x \\
= & \frac{(n-2)}{n} \frac{\operatorname{Cap}(K)}{n(n-2) \omega_{n}|K|^{\frac{n-2}{n}}-1} \int_{\Sigma}\langle X, \eta\rangle \mathrm{d} \sigma \\
& +\frac{1}{n(n-2) \omega_{n}|K|^{\frac{n-2}{n}}}\left[2 \int_{\Omega}\left\langle\nabla U, \nabla \frac{\partial U}{\partial t}\right\rangle \mathrm{d} x+\int_{\Sigma}\langle X, \eta\rangle|\nabla U|^{2} \mathrm{~d} \sigma\right] \\
= & \frac{(n-2)}{n} \frac{\operatorname{Cap}(K)}{n(n-2) \omega_{n}|K|^{\frac{n-2}{n}}-1} \int_{\Sigma}\langle X, \eta\rangle \mathrm{d} \sigma \\
& +\frac{1}{n(n-2) \omega_{n}|K|^{\frac{n-2}{n}}}\left[\int_{\Sigma}\langle X, \eta\rangle|\nabla U|^{2} \mathrm{~d} \sigma-2 \int_{\Omega} \frac{\partial U}{\partial t} \Delta U \mathrm{~d} x\right.
\end{aligned}
$$




$$
\begin{aligned}
& \left.+2 \int_{\Sigma} \frac{\partial U}{\partial t} \frac{\partial U}{\partial \eta}\right] \\
= & \frac{(n-2)}{n} \frac{\operatorname{Cap}(K)}{n(n-2) \omega_{n}|K|^{\frac{n-2}{n}-1}} \int_{\Sigma}\langle X, \eta\rangle \mathrm{d} \sigma \\
& -\frac{1}{n(n-2) \omega_{n}|K|^{\frac{n-2}{n}}}\left[\int_{\Sigma}\langle X, \eta\rangle\left(\frac{\partial U}{\partial \eta}\right)^{2} \mathrm{~d} \sigma\right] \\
= & \frac{1}{n(n-2) \omega_{n}|K|^{\frac{n-2}{n}}}\left[\frac{(n-2)^{2} \omega_{n} \operatorname{Cap}(K)}{|K|} \int_{\Sigma}\langle X, \eta\rangle \mathrm{d} \sigma\right. \\
& \left.-\int_{\Sigma}\langle X, \eta\rangle\left(\frac{\partial U}{\partial \eta}\right)^{2} \mathrm{~d} \sigma\right] .
\end{aligned}
$$

Here we have used the fact that $U$ is constant on $\Sigma$, so $|\nabla U|=\frac{\partial U}{\partial \eta}$ there.

Corollary 7 A compact set $K$ with nonempty interior $\mathcal{O}$ and smooth boundary $\Sigma$ is a critical point of $\mathcal{E}_{0}$ if and only if $\Omega=\mathbb{R}^{n} \backslash K$ supports a solution to the over-determined boundary value problem (1.3).

\section{Proof Setting}

$$
\Lambda^{2}=\frac{(n-2)^{2} \omega_{n} \operatorname{Cap}(K)}{|K|},
$$

we use (A.3) to see that $K$ is a critical point of $\mathcal{E}_{0}$ if and only if

$$
\int_{\Sigma}\langle X, \eta\rangle\left[\Lambda^{2}-\left(\frac{\partial U}{\partial \eta}\right)^{2}\right] \mathrm{d} \sigma=0
$$

for all possible variation fields $X$, which in turn implies

$$
\frac{\partial U}{\partial \eta}=\Lambda=(n-2) \sqrt{\frac{\omega_{n} \operatorname{Cap}(K)}{|K|}}
$$

along $\Sigma$. We notice that if $K=\overline{\mathbf{B}}_{\rho}$ this constant reduces to $\Lambda=\frac{n-2}{\rho}$.

Conversely, let $\Omega$ admit a solution to (1.3). By the uniqueness of solutions to (1.2), this function must be the equilibrium potential of $K$, so by (A.3)

$$
\left.\frac{\mathrm{d}}{\mathrm{d} t} \mathcal{E}_{0}\left(K_{t}\right)\right|_{t=0}=0
$$

for all possible variation fields $X$.

Though we will not use it, we include the following derivation to satisfy the reader's curiousity. 
Lemma A.2 We have

$$
\begin{aligned}
& -\left.\frac{\mathrm{d}}{\mathrm{d} t} \mathcal{E}_{1}\right|_{t=0}=\frac{1}{|\Sigma|^{\frac{n-2}{n-1}}}\left[\left(\frac{n-2}{n-1}\right)\left(\frac{\operatorname{Cap}(K)}{|\Sigma|}\right) \int_{\Sigma}\langle X, \eta\rangle H_{\Sigma} \mathrm{d} \sigma\right. \\
& \left.+\frac{1}{n(n-2) \omega_{n}} \int_{\Sigma}\langle X, \eta\rangle\left(\frac{\partial U}{\partial \eta}\right)^{2} \mathrm{~d} \sigma\right] .
\end{aligned}
$$

Proof We begin by differentiating the boundary condition $\left.U\right|_{\Sigma}=1$ to see

$$
\frac{\partial U}{\partial t}=-\langle X, \eta\rangle \frac{\partial U}{\partial \eta}
$$

on $\Sigma$. Thus

$$
\begin{aligned}
& \left.\frac{\mathrm{d}}{\mathrm{d} t} \mathcal{E}_{1}\right|_{t=0}=\left.\frac{\mathrm{d}}{\mathrm{d} t}\right|_{t=0}\left(\frac{1}{n(n-2) \omega_{n}}|\Sigma|^{\frac{2-n}{n-1}} \int_{\Omega_{t}}\left\langle\nabla U_{t}, \nabla U_{t}\right\rangle \mathrm{d} x\right) \\
& =\frac{1}{|\Sigma|^{\frac{n-2}{n-1}}}\left(\frac{2-n}{n-1}\right)|\Sigma|^{-1} \frac{1}{n(n-2) \omega_{n}} \int_{\Omega}|\nabla U|^{2} \mathrm{~d} x \int_{\Sigma}\langle X, \eta\rangle H_{\Sigma} \mathrm{d} \sigma \\
& +\frac{1}{n(n-2) \omega_{n}|\Sigma|^{\frac{n-2}{n-1}}}\left[2 \int_{\Omega}\left\langle\nabla U, \nabla \frac{\partial U}{\partial t}\right\rangle \mathrm{d} x+\int_{\Sigma}\langle X, \eta\rangle|\nabla U|^{2} \mathrm{~d} \sigma\right] \\
& =-\left(\frac{n-2}{n-1}\right) \frac{\operatorname{Cap}(K)}{|\Sigma|^{\frac{n-2}{n-1}+1}} \int_{\Sigma}\langle X, \eta\rangle H_{\Sigma} \mathrm{d} \sigma \\
& +\frac{1}{n(n-2) \omega_{n}|\Sigma|^{\frac{n-2}{n-1}}}\left[\int_{\Sigma}\langle X, \eta\rangle|\nabla U|^{2} \mathrm{~d} \sigma-2 \int_{\Omega} \frac{\partial U}{\partial t} \Delta_{0} U \mathrm{~d} x\right. \\
& \left.+2 \int_{\Sigma} \frac{\partial U}{\partial t} \frac{\partial U}{\partial \eta} \mathrm{d} \sigma\right] \\
& =-\frac{1}{|\Sigma|^{\frac{n-2}{n-1}}}\left[\left(\frac{n-2}{n-1}\right) \frac{\operatorname{Cap}(K)}{|\Sigma|} \int_{\Sigma}\langle X, \eta\rangle H_{\Sigma} \mathrm{d} \sigma\right. \\
& \left.+\frac{1}{n(n-2) \omega_{n}} \int_{\Sigma}\langle X, \eta\rangle\left(\frac{\partial U}{\partial \eta}\right)^{2} \mathrm{~d} \sigma\right] \text {. }
\end{aligned}
$$
Here we've used the fact that $H_{\Sigma}$ is the first variation of $|\Sigma|$ and that $|\nabla U|=-\frac{\partial U}{\partial \eta}$
on $\Sigma$.

One can mimic the proof of Corollary 7 to show that critical points of $\mathcal{E}_{1}$ are precisely those sets $K$ which admit a solution to the over-determined boundary value problem

$$
\Delta U=0,\left.\quad U\right|_{\partial K}=1, \quad \lim _{|x| \rightarrow \infty} U(x)=0, \quad \frac{\partial U}{\partial \eta}=\Lambda H,
$$

where $H$ is the mean curvature of $\Sigma=\partial K$. 


\section{References}

1. Bray, H.: Proof of the Riemannian Penrose inequality using the positive mass theorem. J. Differ. Geom. 59, 177-267 (2001)

2. Eichmair, M., Metzger, J.: Unique isoperimetric foliations of asymptotically flat ends in all dimensions. Invent. Math. 194, 591-630 (2013)

3. Fall, M.M., Minlend, I.A.: Serrin's over-determined problem in Riemannian manifolds. Adv. Calc. Var. 8, 371-400 (2015)

4. Goldman, M., Novaga, M., Ruffini, B.: Existence and stability for a non-local isoperimetric model of charges liquid drops. Arch. Ration. Mech. Anal. 217, 1-36 (2015)

5. Huisken, G., Yau, S.-T.: Definition of center of mass for isolated physical systems and unique foliations by stable spheres with constant mean curvature. Invent. Math. 124, 281-311 (1996)

6. Jauregui, J.: ADM mass and the capacity-volume inequality at infinity. arxiv:2002.08941

7. Lamm, T., Metzger, J., Schulze, F.: Foliations of asymptotically flat manifolds by surfaces of Willmore type. Math. Ann. 350, 1-78 (2011)

8. Metzger, J.: Foliations. of asymptotically flat 3-manifolds by 2-surfaces of prescribed mean curvature. J. Differ. Geom. 77 (2007), 201-236

9. Pacard, F., Rivière, T.: Linear and Nonlinear Aspects of Vortices: The Ginzburg-Landau Model. Birkhäuser, Basel (2000)

10. Raulot, S., Savo, A.: On the spectrum of the Dirichlet-to-Neumann operator acting on forms of a Euclidean domain. J. Geom. Phys. 77, 1-12 (2014)

11. Schoen, R., Yau, S.-T.: Conformally flat manifold, Kleinian groups, and scalar curvature. Invent. Math. 92, 47-71 (1988)

12. Serrin, J.: A symmetry problem in potential theory. Arch. Ration. Mech. Anal. 43, 304-318 (1971)

13. Struwe, M.: Variational Methods: Applications to Nonlinear Partial Differential Equations and Hamiltonian Systems. Springer, Berlin (2008)

14. Ye, R.: Foliation by constant mean curvature spheres on asymptotically flat manifolds. In: Geometric Analysis and the Calculus of Variations, pp. 369-383. International Press, Cambridge (1996)

Publisher's Note Springer Nature remains neutral with regard to jurisdictional claims in published maps and institutional affiliations. 